\title{
Das Gedächtnis der Kulturwis- senschaften und die Klassische Tradition: Erinnern und Vergessen im Licht interdisziplinärer Forschung
}

\author{
DIETRICH HARTH
}

Memory and forgetting are key subjects of current historical and sociological research. Their meaning embraces passive as well as active forms of collective recollection and oblivion, activities of retention as well as of dynamic shifting in the broad field of symbolic representation. Applied to Rezeptionsgeschichte and Wirkungsgeschichte in the perspective of the Classical Tradition, those key terms signify the complex interplay between change and continuity. The materials discussed under this assumption mainly range from theories and practices of memory in Antiquity and the Middle Ages to Renaissance systems of architecture and art, from the early emergence of advanced civilizations to a modern conceptualized history of cultural change. The connecting element is the theoretically defined concept of "cultural memory" not only comprising textuality and literary techniques of memory but also image production and ritual activities in non-literate societies. At the end the question will be asked, whether there are any alternative ways of cultureinvestigation lying hidden within cultural memory itself.

... nam et omnis disciplina memoria constat.

Quintilian

$\mathrm{D}$ ie Altertumsforschung der letzten Jahre wendet sich mit zunehmender Intensität den Perspektiven und Interessen zu, die der Rezeption, ja 'Konstruktion' der antiken Literatur und Kultur zugrunde liegen. ${ }^{1}$ Die folgende Studie geht anhand eini-

1. Vgl. z.B. folgende Sammelbände: R. Herzog, R. Koselleck (Hg.): Epochenschwelle und Ephochenbewußtsein, München 1987 (hier insbes. die Beiträge von M. Fuhrmann, R. Herzog, C. Meier); W. Voßkamp (Hg.): Normativität und Historizität europäischer Klassiken, Stuttgart/Weimar 1993; H. Flashar (Hg.): Altertumswissenschaft in den 20er Jahren. Neue Fragen und Impulse, Stuttgart 1995.

Dietrich Harth, Universität Heidelberg, Germanistisches Seminar, Hauptstraße 207-209, D-69117 Heidelberg, Germany. 
ger ausgewählter Neuerscheinungen den theoretischen und methodologischen Voraussetzungen nach, von denen diese Forschungsrichtung sich leiten läßt. Sie versteht sich nicht ausschließlich als Forschungsbericht, sondern versucht, Gewinn und Verlust abzuwägen und Alternativen wenigstens anzudeuten. Die meisten der ausgewählten Arbeiten beziehen sich auf antike Quellen und deren kontextabhängigen Funktionswandel, wenige auf die Praktiken kollektiven Erinnerns in außereuropäischen Kulturen. Diese zuletztgenannten Studien bieten Gelegenheit, auf mögliche Grenzüberschreitungen zwischen Kulturgeschichte und Kulturanthropologie aufmerksam zu machen.

\section{"The past is a foreign country"}

Wie zahlreiche andere begriffliche Münzen im okzidentalen Gedankenkommerz zeigen "Erinnerung" und "Gedächtnis" - reminiscentia et memoria - unterm Vergrößerungsglas der historischen Semasiologie Spuren ältester Prägung. Es ist nicht überraschend, daß früh schon das Gedächtnis als eine Erfindung (inventio) des anthropologischen Diskurses und als ein von der Natur dem tierischen Organismus eingepflanztes Orientierungsvermögen verstanden worden ist. Der Begriff - nicht nur der deutschsprachige - evoziert beides: das Denken (Subjekt) und das Gedachte (Objekt). "Schon unsere Sprache gibt dem Gedächtnis", schrieb Hegel, "die hohe Stellung der unmittelbaren Verwandtschaft mit dem Gedanken."2

Aristoteles nannte "Gedächtnis" (mnéme) ein elementares, naturwüchsiges Merkvermögen, "Erinnerung" (anámnesis) eine allein dem menschlichen Bewußtsein eigene, den Zeitsinn einschließende kognitive Reproduktions- und Retentionskraft. Beide durch gemeinsame Grenzen vereinte Konzeptionen, über deren intrikates Verhältnis noch heute - in Psycho- und Neuro-Wissenschaften unter empirischen Vorzeichen - gerätselt wird, ${ }^{3}$ unterscheiden sich indessen von einer älteren, in mythologischen, poetischen, hermetischen Symbol- und Bildkomplexen aufbewahrten Überlieferung. Die in diesem Kontext auftretenden Zeichen und Bilder zielen zum einen auf das Vermögen der Anamnese, das gleichsam archäologische Ausgraben latenten Wissens, zum andern - unter dem Namen Mnemosyne - auf ein produktives, von der Einbildungskraft (imaginatio) unterstütztes Vermögen, früher Erlebtes im Licht späterer Erfahrungen um- bzw. neuzugestalten. Mnemosyne ist nicht nur die Mutter der Historiographie (Klio), sondern aller symbol-, bild- und zeichenträchtigen Künste. Erst das Zusammenspiel von Anamnese und Mnemosyne legt es nahe, im absichtsvollen Akt der re-cordatio einen Akt der Bedeutungsbildung zu sehen, der sich bewußt auf gegebenes Wissen und kulturelle Artefakte zurückbeugt. Das berührt die formalen Bedingungen kultureller Prozesse, die ich unter dem Begriff der Rekursivität zusammenfassen möchte: Jedes kulturelle System bildet im Verlauf seiner Entstehung ein Inventar standardisierter, materiell verfügbarer Elemente und Normen aus, das es erlaubt, eine schier unendliche Menge variabler, gleichwohl einem relativ einheitlichen Kulturstil entsprechender Muster zu entwickeln.

2. G. W. F. Hegel: Enzyklopädie der philosophischen Wissenschaften, § 464. - Zum Funktions- und Bedeutungswandel der thematischen Konzepte vgl. die von mir hrsg. Anthologie Die Erfindung des Gedächtnisses (Frankfurt/M. 1991) und meinen Artikel "Erinnerung \& Gedächtnis", in: Handbuch Historische Anthropologie, hg.v. C. Wulf (erscheint 1996).

3. I. Rosenfield: The Invention of Memory. A New View of the Brain, New York 1988. 
Es ist aber angebracht, das spontane (gelebte) mit dem hier so genannten anamnetischen (historischen) Erinnern nicht zu verwechseln und dementsprechend das persönliche von jenem mnemotechnischen Erinnern $\mathrm{zu}$ unterscheiden, das im Sinne institutionalisierter, öffentlich anerkannter Regulierinstanzen das Zusammenspiel von Anamnese und Mnemosyne kontrolliert. "Mnemotechnik" ist in dem für mein Vorhaben abgesteckten Diskussionsrahmen auf die instrumentellen Funktionen der rhetorischen Ars memorativa, die der Begriff gewiß auch umfaßt, nicht zu reduzieren. Der Begriff steht hier für verschiedene Formen der gruppen- oder gemeinschaftsbezogenen Gedächtnisorganisation: vom Opferritual bis zur institutionalisierten Textauslegung.

Mnemotechnisch Erinnertes bleibt indessen nicht selten - wie die deutsche Sprache sagt - "auswendig", also der subjektiven Erfahrung äußerlich, und verdankt sich einem Prozeß gezielten Lernens, als dessen Agent das funktionale Gedächtnis gilt. Dieses operiert zweckrational, indem es das Zufällige der mnemonischen Bedeutungsbildung methodisch unter Kontrolle zu bringen sucht. Es reguliert die Auswahl der Gedächtnisinhalte nach dem Axiom maximalen Nutzens und schließt somit als überflüssiges Wissen aus, was sich nicht unter die Logik zweckrationaler Entscheidungen fügt. Alternative Gedächtnisinhalte bleiben ausgeschlossen. Die Macht dieses Gedächtnistypus ist nicht zuletzt an dem universalistischen Anspruch solcher Rationalitätsmodelle abzulesen, die mit den Gesetzeswissenschaften identisch sind. Anders das narrative Gedächtnis, das auf partikulare und kontingente Erfahrungen zurückblickt, um diese verstehend in den Horizont der Gegenwart einzuholen. Wer in diesem Rahmen bewußt erinnert, versenkt sich suchend in die Überbleibsel vergangener Welten, ohne je gewiß sein zu können, was an der Geschichte, die das Latente erzählend heraufbeschwört, zur Wahrheit, was zur Legende gehört. Dieser Gedächtnistyp kennt keine festen Garantien und ist daher prinzipiell offen für Fehlleistungen des Erinnerns und unterdrückte Gedächtnisalternativen.

Wer zu Beginn der Neuzeit unter der Devise "antiquatio theoriarum" (P. Rossi) die Abhängigkeit des Denkens und Tuns vom überlieferten Wissenskanon aufheben wollte, um gegebene Wahrheiten nicht zu finden, sondern aufgegebene Wahrheiten erst zu entdecken, trat als Parteigänger jener ars oblivionis auf, deren Erkennungszeichen die tabula rasa ist, das radierte, in jungfräuliche Unschuld zurückverwandelte, das 'leere' Gedächtnis. ${ }^{4}$ Ein Verfahren des Ausschließens, das den modernen Wissenschaftsrevolutionen zur Regel wurde, ohne daß dies den Nutzen des Überlieferten als frei zu variierende kritische Kontrafaktur einer situationistischen Haltung geschmälert hätte. ${ }^{5}$ So hat die zukunftsorientierte Neuzeit auf empirisch-wissenschaftlicher wie traditionskritischer Ebene zwar die autoritative Funktion der gelebten Tradition als Erinnerungsmedium geschwächt. Das diesem Prozeß zu verdankende Geschichtsbewußtsein hat jedoch - in der institutionellen Gestalt der historisch-philologischen Gelehrsamkeit das Alte in Archiv, Bibliothek und Museum konserviert. Es hat auf diese Weise und unter Bedingungen, die noch zu nennen sind, ein verwaltbares wissenschaftliches Gedächtnis geschaffen, das den Modus der Reflexion einsetzt, um das Gedächtnis wirkender Traditionen in ein Aggregat objektiver Geschichten umzuwandeln, das jedoch prinzipiell

4. P. Rossi: "La scienza e la dimenticanza", Iride VIII/14 (1995), S.157, zitiert u.a. Francis Bacon: "Scientia ex naturae lumine petenda, non ex antiquitatis obscuritate repetenda est. Nec refert quid factum fuerit. Illud videndum quid fieri possit."

5. P. Rossi: Il passato, la memoria, l'oblio. Sei saggi di storia delle idee, Bologna 1991, S.155ff. 
für Um-Schreibungen offen steht. ${ }^{6}$ Es ist trivial, aber die unablässig fortschreitende Arbeitsteilung und Spezialisierung in den modernen kulturwissenschaftlichen Disziplinen hat die Kontexte der Erinnerungs- und Gedächtnisbegriffe vervielfacht. Zwar erschwert diese Entwicklung die Übersicht, sie eröffnet aber die Chance, die historische Erinnerungsarbeit als ein Operationsfeld semantischer Konstruktionen ausführlicher als bisher theoretisch zu explizieren.

Die Dialektik von Erinnern und Vergessen muß der bewahrenden und interpretierenden, sprich: der historisch-philologischen Gedächtnispflege von ihren frühesten Anfängen an vertraut gewesen sein. Denn schon die erste, im Auftrag eines Herrschers gegründete Bibliothek war nichts anderes als ein mnemotechnischer Apparat der Bewahrung, an den je verschiedene Kanonstiftungen anknüpfen konnten. Daran hat die Verwissenschaftlichung zunächst nur wenig geändert. Denn die Auswahl, Verwaltung und Auslegung des Traditionswissens und die im 19. Jahrhundert vorangetriebene Professionalisierung der Erinnerungsarbeit standen im Dienst nationaler Identitäten. ${ }^{7}$ Die dort geübte Kanonbildung hat nicht selten eine puristische, ja exklusiv-feindselige Politik verfolgt und in die Archive des Vergessens verbannt, was nicht zum normativen Richtmaß der Modernisierung passen wollte. Doch wie so oft in der Geschichte hat auch dieser Akt kultureller Normierung einen potenten Gegner ins Leben gerufen: die Kritik des falschen bzw. verzerrten, über sich selbst getäuschten Bildungsbewußtseins. ${ }^{8}$ Diese Kritik brachte in unserm Jahrhundert - gegen manchen Widerstand - allmählich eine Forschung in Gang, die sich mehr und mehr der Kehrseite des Erinnerns, dem natürlichen Vergessen, nach und nach auch dem pathologischen Schein eines hegemonialen kulturellen Gedächtnisses und zugleich damit den vielfältigen Formen kollektiver Verdrängung zugewandt hat. Dieser Wandel hat die Archive des Vergessens geöffnet und das Volumen der wissenschaftlich organisierten Denkmalspflege um die Phänomene der gelebten Erinnerung - Alltag, privates Leben, Mentalitäten, Minderheiten- und Randgruppenkulturen - erweitert. Irgendwo - so lautet die Annahme - hat das, was vergessen schien, eine Spur hinterlassen, die der Historiker als geschulter Spurenleser wieder ins Gedächtnis rufen kann. Die Vergangenheit ist ein fremdes Land, eine virtuelle Realität unterhalb der Verdrängungsschwelle. $^{9}$

Die steigende Konjunktur globaler Migrationsbewegungen und des digitalisierten

6. "Wissenschaftliches Gedächtnis" verwende ich in der Bedeutung von "kontrapräsentischer Erinnerung" nach G. Theissen: "Tradition und Entscheidung. Der Beitrag des biblischen Glaubens zum kulturellen Gedächtnis", in: Kultur und Gedächtnis, hg.v. J. Assmann / T. Hölscher, Frankfurt/Main 1988. Theissen spricht zwar die "kontrapräsentische" Funktion dem zu, was er das "kulturelle Gedächtnis" nennt: "Kulturelles Gedächtnis hält kontrapräsentisch fest, was ohne die bewußte Anstrengung der Erinnerung verlorenginge. Das von ihm Erinnerte muß nicht aktuell sein, kann aber immer wieder aktuell werden" (S.171). Diese Beschreibung möchte ich aber nur für die Art der Gedächtnisbewahrung gelten lassen, die im Sinne des historischen Bewußtseins den Zeitenabstand zwischen Vergangenheit und Gegenwart reflektiert.

7. Hinweise auf die kultur-bzw. sprachwissenschaftlichen Beiträge zur 'Erfindung' von Nationalismen bei B. Anderson: Imagined Communities. Reflections on the Origin and Spread of Nationalism, London 1983.

8. Vgl. zur Geschichte dieses Deutungsmusters die Untersuchung von G. Bollenbeck: Bildung und Kultur. Glanz und Elend eines deutschen Deutungsmusters, Frankfurt/Main 1994.

9. The past is a foreign country lautet der Titel eines Buches von David Lowenthal (Cambridge 1985). 
intra- wie transkulturellen Informationsaustauschs verändert indes das traditionelle Selbstverständnis der historisch verfahrenden Archiv- und Inventarwissenschaften. Nicht die eine geschlossene, reine und in sich ruhende Kultur - ohnehin eine Fiktion soll ihr nun als einziges Richtmaß gelten, sondern das zusammengesetzte, grenzüberschreitende, im fließenden Übergang zwischen Grenze und Zentrum sich konsolidierende und zugleich dekomponierende fragile Gebilde symbolischer Repräsentationen. Die Lage ist paradox. Denn nicht nur die Kulturwissenschaften, auch die lebensweltlichen Erfahrungen, ihr eigentliches Fundament, scheinen heute stärker denn je die von der "Furie des Verschwindens" (Hegel) verschuldete kollektive Amnesie zu fürchten. Die jüngsten und aktuellen Erfahrungen der Weltkriege, der Vertreibungen, des atomaren Holocaust, des Völkermordes, der Kommunikationsentropie, des linguistischen und biologischen Artenschwunds etc. sind die Kehrseite der oben skizzierten Entwicklung. Nicht alles ist friedlicher, Ressourcen und Traditionen schonender Umbau, nicht weniges Wandel durch Gewalt und Zerstörung. ${ }^{10}$ Auch die Konsequenzen sind paradox: einerseits eine fast schrankenlose Kommunikation zwischen Gesellschaften und Kulturen, andererseits eine planvoll betriebene Exterminierung ganzer Völker; einerseits das schnelle Verschwinden traditionaler Lebensformen, andererseits deren nicht weniger rasche Kartierung in immer subtiler ausgetüftelten künstlichen Gedächtnissen. Eins wie das andere berührt die beschränkte Kapazität des Gedächtnisses und das selektive Spiel der Erinnerung. Erfahrungen, die gemeinsam mit andern, weniger offensichtlichen Motiven für die aktuelle Konjunktur des wissenschaftlichen Gedächtnisdiskurses verantwortlich sind. ${ }^{11}$

"Gedächtnis" und "Erinnerung" sind vor allem im interdisziplinären Wissenschaftsdiskurs längst zu Leitmotiven der historischen Forschung geworden. Auch in jener fächerübergreifenden Disziplin, die sich der Anamnese des Unvordenklichen ("anamnesi dell'immemorabile") verschrieben hat, in der Philosophie, feiern altehrwürdige Topoi des Gedächtnisdiskurses sonderbare Urständ. ${ }^{12}$ Die wissenschaftlichen Fragen haben sich jedoch vom Inhalt und Kanon auf Methode und Prozeß verschoben: Nicht nur was ins Gedächtnis gehört, sondern wie erinnert wurde und wird, beschäftigt in zunehmendem Maß die methodisch disziplinierte Neugier. Die mannigfachen Hinterlassenschaften zu konservieren, ist eine Sache; eine andere, ob und wie sie in lebendige Erinnerung übergehen können.

Vor diesem Hintergrund möchten die folgenden Betrachtungen gelesen werden. Sie gehen zunächst - in der Art kurzer Fallstudien - anhand einzelner Neuerscheinun-

10. Zur legitimatorischen Funktion kultureller Muster für die Anwendung und Aufrechterhaltung physischer und struktureller Gewalt vgl. den systematischen Aufriß von J. Galtung: "Cultural violence", Journal of Peace Research $27 / 3$ (1990), S.291-305, und den Beitrag von A. und J. Assman: "Kultur und Konflikt. Aspekte einer Theorie des unkommunikativen Handelns", in: J. Assmann / D. Harth (Hg.): Kultur und Konflikt, Frankfurt/Main 1990, S.11-48.

11. Vgl. auch den interessanten, vom Generationenabstand ausgehenden Erklärungsversuch J. Assmanns in seiner unten genannten Untersuchung (Anm.13, S.21ff.).

12. "Quali interrogativi la scienza pone alla filosofia? Conversazione con Massimo Cacciari", in: P. Alferi / A. Pilati (Hg.): Conoscenza e complessità, Rom/Neapel 1990, S.164. Zur permanenten Um-Schreibung des kulturellen Gedächtnisses in der modernen Philosophie vgl. den Sammelband Philosophical Imagination and Cultural Memory. Appropriating Historical Traditions (hg.v. P. Cook, Durham/London 1993), dessen Titel und Einleitung hinter der nützlichen, von Theissen (Anm.6) vorgeschlagenen Unterscheidung zwischen "Tradition" und "kulturellem Gedächtnis" zurückbleibt. 
gen den Methoden und Befunden der Gedächtnisforschung in verschiedenen kulturwissenschaftlichen Disziplinen nach. ${ }^{13}$ Am Schluß kehren sie noch einmal zu den hier angedeuteten Problemen zurück, um der Vermutung Raum zu geben, daß sich hinter der Konjunktur des Gedächtnisbegriffs in den Kulturwissenschaften ein altes Dilemma verbirgt.

\section{"Ancient and medieval memories set the agenda for modernity"}

Frances A.Yates hat ihre berühmte Untersuchung The Art of Memory (1966) der rhetorischen Gedächtniskunst und ihrer bis zur Geburt der Neuen Wissenschaften im 17. Jahrhundert andauernden lebendigen Modellierung gewidmet. Thr Ausgangspunkt war die Hermetische Philosophie Giordano Brunos, methodisch nahm sie Anregungen C. G. Jungs auf und konnte an die philosophiegeschichtlichen Untersuchungen Paolo Rossis über die Artes memorativae in Renaissance und Barock anknüpfen. ${ }^{14}$ Ein Vierteljahrhundert später gelten andere Auslegungsparadigmen. Heute läßt sich die historisch-philologische Gedächtnisforschung von anderen Göttern inspirieren, nimmt in zunehmendem Maß Anregungen aus Medizin und Naturwissenschaften auf. ${ }^{15}$

Janet Colemans umfangreiche und informative Studien, 1992 unter dem Titel Ancient and medieval memories veröffentlicht, sind eine Antwort auf diesen Paradigmenwechsel. Sie beabsichtigt, mit der Rekonstruktion komplexer, in Altertum und Mittelalter entworfener Theorien und Praktiken den materialistischen und mechanistischen Reduktionen der messenden und experimentierenden Wissenschaften entgegenzutreten (SS. XVf. u. 593ff.): ein gegenwartskritisches Motiv der historischen Arbeit, das alte und älteste Problemlagen in Erinnerung ruft, um die Theoriebildung von einem Standort aus zu provozieren, dem nur eine interdisziplinäre Debatte gerecht werden kann.

Colemans detaillierte Darstellung, deren Gehalt hier nur in knapper Form wiedergegeben werden kann, ist im besten Wortsinn problemgeschichtlich, enzyklopädisch im Hinblick auf die behandelte Zeitspanne (von Platon bis Hobbes), verfährt hermeneutisch sowie kulturgeschichtlich und ist insofern - wie die Verfasserin selber bemerkt (S.XIII) - interdisziplinär. Die Dispositio des Buches folgt dem Gebot der narrativen Diachronie, und es erscheint mir sinnvoll, statt langatmiger Umschreibungen an dieser Stelle den wie ein klassisches Drama in fünf Akte gegliederten Aufbau der Untersuchung zu zitieren:

\section{Part I The critical texts of antiquity}

Part II The practice of memory during the period of transition from classical antiquity to the christian monastic centuries

Part III The beginnings of the scholastic understanding of memory

13. Im Zentrum stehen folgende Publikationen: J. Coleman: Ancient and medieval memories. Studies in the reconstruction of the past, Cambridge 1992; M. Carpo: Metodo e ordini nella teoria architettonica dei primi moderni: Alberti, Raffaello, Serlio e Camillo, Genf 1993; S. Küchler / W. Melion (Hg.): Images of memory. On remembering and representation, Washington/London 1991; J. Assmann: Das kulturelle Gedächtnis. Schrift, Erinnerung und politische Identität in frïhen Hochkulturen, München 1992.

14. Vgl. insbesondere P. Rossi: Clavis Universalis. Arti mnemoniche e logica combinatoria da Lullo a Leibniz, Mailand/Neapel 1960.

15. Das gilt nicht nur für Colemans Studien, sondern auch für die Untersuchung von Mary J. Carruthers: The Book of Memory. A Study of Memory in Medieval Culture, Cambridge 1990. 


\section{Part IV Aristotle neo-Platonised: The revival of Aristotle and the development of scholastic theories of memory \\ Part $V$ Later medieval theories of memory: The via antiqua and the via moderna}

Die Überschriften der Unterkapitel nennen meist die Namen der interpretierten Autoren; in Teil I z.B. Platon, Aristoteles, Cicero, Plotin, Augustinus. Diese Art der Gliederung empfiehlt das Buch, zusammen mit einem ausführlichen Index, auch als Nachschlagewerk; andere Autoren mit eigenen Kapiteln sind Gregor der Große, St. Benedikt, St. Bernhard, Beda, Anselm, Abelard, John of Salisbury, John Blund, Averroes, Albertus Magnus, Thomas von Aquin, Duns Scotus, William of Ockham, Petrarca. Die Namen deuten an, daß es in diesem Buch vor allem um theoretische Fragen geht. Eine Besonderheit der Darstellung sind die (gleichsam als Motti zahlreichen Kapiteln vorangestellten) langen Zitate aus der modernen poetischen und wissenschaftlichen Literatur. Hier finden sich neben Shakespeare-Zitaten Auszüge aus Proust, Luis Buñuel, Gertrude Stein und nicht zuletzt zahlreiche Textstellen aus der Literatur zur Neurobiologie, Psycholinguistik, Kognitions- und Experimentalpsychologie. Es entsteht so ein moderner Subtext aus Fragmenten, an dessen Aussagen der Leser, wenn er mag, Analogien und Differenzen zum dargelegten historischen Problemstand ablesen kann.

Das erste Kapitel des dritten Teils ist Abelard überschrieben. Mit dessen Nominalismus nimmt die traditionelle Theoriebildung, was die Zentralstellung dieses Teils in der Gesamtkomposition des Buches unterstreicht, eine auf die Moderne vorausweisende Wende: Abelard - schreibt Coleman (S.232) - bemühte sich als einer der ersten um eine "Theorie historischen Verstehens". Diese Aussage schlägt das auch im Untertitel des Buches genannte Leitthema an: die Funktion des Gedächtnisses in der methodischen Rekonstruktion der Vergangenheit.

Coleman hat dieses Thema $\mathrm{zu}$ ihrem eigenen Verfahrensprinzip gemacht. Sie rekonstruiert nicht nur, sondern legt eine Tradition frei, von der sie überzeugt ist, daß sie zum verborgenen kulturellen Gedächtnis der Moderne gehört. Couragiert formuliert sie daher als Leitmotiv: "Ancient and medieval memories set the agenda for modernity" (S. 541). Moderne Psychologie und Hirnforschung haben erkannt, daß am Prozeß des Erinnerns nicht nur eine isolierbare Hirnregion, sondern alle mentalen Funktionen beteiligt sind. Coleman folgt dieser Einsicht, vermeidet aber die simplifizierende Gleichsetzung von Gehirn (brain) und Geist (mind). Ihr Interesse ist vor allem epistemologischer, dann erst geschichtstheoretischer Natur, und sie konzentriert sich daher meist auf die spekulativen Kontexte der anamnetischen (methodischen) Gedächtnisarbeit (reminiscence), ohne die Grenzziehung zwischen dieser und dem spontanen Erinnern (remembering) zu vernachlässigen.

Es sind vorab drei Schlüsseldiskurse des griechischen und lateinischen Altertums, die von den Theoretikern wie Praktikern der methodischen Gedächtnisarbeit in Spätantike und Mittelalter immer wieder aufgegriffen und hin und her gewendet worden sind:

Erstens: Platons Kritik an der technisch (rhetorisch) und zeichenhaft (schriftlich) fundierten Erinnerung, ein notdürftiges Erinnern, das in Wahrheit das Vergessen jener reinen Formen des Seins, der Ideen, zur Folge hat, die allein der Anamnese zugänglich sind.

Zweitens: Aristoteles' Unterscheidung zwischen der interpretierenden, da- 
her wahrheitsfähigen, rhetorisch bzw. poetisch gestalteten Vergangenheitskunde auf der einen und der kontingenten Darstellung der gelebten Vergangenheit in der Historiografie auf der anderen Seite. Eine Position, die ausdrücklich die dem Gedächtnis eingeprägte materielle ikonische Spur der vergangenen Erfahrung gegen Platons Kritik verteidigt und das Erinnern mit rationalen Operationen verbindet.

Drittens: Die Rhetorik als pragmatisches Organon der Textproduktion und der Mnemotechnik, ein die Vergangenheitskunde und -darstellung ( $h i$ storia) modellierendes und lange Zeit beherrschendes Lehr- und Lernsystem. ${ }^{16}$ Nicht zu vergessen die in diesem Diskurs kultivierte erkenntnistheoretische Skepsis, für die der Name Ciceros gleichsam das Codewort liefert.

Diese drei Schlüsseldiskurse haben, wie Colemans textnahe Analysen zeigen, über die patristische Rezeption Eingang in die christlichen Gedächtnis- und Geschichtstheorien des Mittelalters gefunden. Für eine Theologie, deren praktische und theoretische Bemühungen einem sakrosankten schriftlichen Kanon verhaftet sind, scheint es unmöglich, Platons Kritik an der schriftgestützten Erinnerung ernst zu nehmen. Und dennoch hat der Neoplatonismus hier eine Brücke konstruiert, die es manchen scholastischen Denkern des Hochmittelalters erlaubte, Aristoteles und Platon zu harmonisieren. Diese Brücke hieß paradoxerweise Vergessen.

Wo immer eine unmittelbare oder mystische Gotteserkenntnis zur Debatte stand, ging es darum, über die 'Leiter' der Wörter, Bilder und Zeichen den Weg in die innere, bild- und zeichenlose Anschauung des Ineffabile zu bahnen. Plotin hat das Gedächtnis nicht als passiven, Eindrücke speichernden Rezeptor verstanden, sondern mit einer seelischen Aktivität identifiziert, die aus eigener Kraft und unter Vergessen der äußerlichen Sinnesobjekte eine "intellektuelle Ordnung" hervorzubringen vermag - " $a$ kind of illuminationism" (S.76ff.). Augustinus hat zwar - im Sinne der rhetorischen Mnemotechnik - das Gedächtnis als Behältnis jener "Bilder" gedeutet, die der Geist von den bestimmten Sinneswahrnehmungen abzieht und nach eigenen Gesetzen mnemonisch ordnet. Aber die unmittelbare, die "spirituelle" Gotteserfahrung knüpfte er an die Bedingung des Selbstvergessens (S.110f.). Es ist vor allem die augustinische, rhetorische, aristotelische, neoplatonische und schriftexegetische Theoreme eklektisch verschmelzende Konzeption, die die Ausbildung des christlichen Gedächtnisdiskurses in den folgenden Jahrhunderten maßsgeblich bestimmen wird.

So praktizieren die monastischen Zentren - die Klöster der Benediktiner und Zisterzienser - eine Kultur des Vergessens und der Gedächtnis-Reinigung, die den sakralen Schriftkanon als einziges grammatisch-rhetorisches Muster und Medium der Gottesannäherung anerkennt. In diesem Rahmen wird die Meditation der Schrift - in der Bedeutung halblauten Lesens - zur maßgebenden Technik des Erinnerns und die Grammatik zum exegetischen Schlüssel, der das Tor zur "göttlichen Weisheit" öffnen soll (S.143). Coleman zeigt, daß die in den Klöstern entstehende frühmittelalterliche Geschichtsschreibung - ein Beispiel ist die Historia ecclesiastica gentis Anglorum (bis 731) Bedas - streng secundum litteram verfuhr. Diese Historie erzählt im Vertrauen auf eine wirklichkeitssubstituierende Kraft des schriftlichen Zeichens, die nicht im modernen Wortsinn das Vergangene als solches 'erinnert', sondern den Leser in den Status des

16. S. meinen Forschungsartikel "Geschichtsschreibung", in: Historisches Wörterbuch der Rhetorik, hg.v. G. Ueding, Tübingen, Bd.III (erscheint 1996). 
gleichzeitigen Augenzeugen versetzen will (S.276ff.) Denn die Buchstaben sind "Anzeichen" des gesprochenen Wortes und der Dinge, die durch die Fenster der Augen und stimmlos zur Seele der Leser 'reden'. ${ }^{17}$ In diesem Kontext ist der Begriff historia identisch mit einer Schreib- und Lesart, die den wörtlichen, den Litteralsinn zur Sprache bringt und daher nur die propädeutische Sprosse auf jener Leiter der Exegese, die in die figurativ-theologischen Stockwerke des mehrfachen Schriftsinnes führt.

Die Verbreitung und Verfeinerung der Schriftkultur und der rationale Umbau des theologischen Lehrsystems im 12. und 13. Jahrhundert verändern den Gedächtnisdiskurs und mit ihm die Historiographie, die in Klöstern und Klerikerschulen bald eine erste Blütezeit erlebt. Coleman erläutert ausführlich die physio- und psychologischen Neuansätze. Das Vergessen erscheint nun den Scholastikern als Verdunkelung der Tradition und "fraudatrix scientiae". ${ }^{18}$ Der nächste Schritt ist die Proklamation der Theologie als selbständige Wissenschaft. Die Sententiae des Petrus Lombardus verdrängen, was Coleman nicht erwähnt, den biblischen Kanon als Grundlagentext für die theologische Spekulation. ${ }^{19}$ Die Herrschaft des neoplatonischen Augustinismus wird - vereinfachend gesagt - von der des aristotelischen Systemdenkens abgelöst. Der christliche Gott ist nicht allein der Liebe, sondern wird nun auch und vor allem der metaphysischen Konstruktion zugänglich.

Im Lauf dieser Entwicklung nehmen Dialektik, Logik und theologische Morallehre den Gedächtnisdiskurs in Besitz, und das historiographische wie theoretische Nachdenken über Zeitsinn und Sinn der Zeit stößt auf die ersten Spuren einer qualitativen Differenz zwischen dem, was ist, und dem, was war. Dennoch kreisen die Bemühungen der meisten Neuerer noch um das alte Problem, wie der - mit Paul Ricoeur zu reden - "Mißklang" zwischen endlicher und kosmischer (Heils-)Zeit zu überwinden ist. ${ }^{20}$ Coleman vertritt die These, die hierzu nötige Vermittlung sei nicht in der Erzählung, sondern in der exegetischen Harmonisierung des Ungleichzeitigen zu suchen. Ungleichzeitig sind nicht nur die Zeugnisse über vergangene und gegenwärtige Ereignisse, Ungleichzeitigkeit prägt auch das Verhältnis zwischen den philosophischen Texten der Antike (an erster Stelle Aristoteles) und dem sakrosankten Kanon der christlichen Überlieferung, dessen zeitresistente Geltung ohnehin nicht in Frage stand.

Unwiederholbarkeit und Einmaligkeit der Ereignisse und ein evolutionärer Zeitrahmen waren für die Theoretiker des Hochmittelalters kein Thema. Deren Vorstellungen entsprachen eher den ritualisierten Zeitformen der Liturgie, waren also zyklischer Natur (S.303). Wenn ein arabischer, von manchen christlichen Theologen geschätzter Aristoteliker wie Avicenna auf die Wechselhaftigkeit und Kontextabhängigkeit des Erinnerns zu sprechen kam (S.361), so hatte das keine Folgen für die Auseinandersetzung mit dem Traditionswissen.

Gewiß, mit der Expansion des Handels und der städtischen Kulturen erwachte allmählich ein Bewußtsein für unterschiedliche Erfahrungswelten und zeitliche Differenzen. Doch in der Theorie blieb die Macht der Synchronie lange noch ungebrochen.

17. Carruthers (Anm.15), S.222ff.

18. John of Salisbury: Policraticus, hg.v. C. C. J. Webb, Oxford 1909, Prolog. Vgl. auch die (von Coleman nicht erwähnte) ausführliche Untersuchung von P. von Moos: Geschichte als Topik. Das rhetorische Exemplum von der Antike zur Neuzeit und die historiae im Policraticus Johanns von Salisbury, Hildesheim 1988.

19. M.-D. Chenu: La Théologie comme science au XIIlème siècle, Paris 1957, SS.15ff., $37 \mathrm{ff}$.

20. P. Ricoeur: "Le temps raconté", Revue de Métaphysique et de Morale 89/4 (1984), S.448. 
Was zeitlich fern und vielleicht fremd erschien, ließ sich mit den theoretischen und praktischen Bedürfnissen der Gegenwart durch die Applikation eines formalen Auslegungsschemas versöhnen, das mit sprachlichen Universalien und Analogieschlüssen operierte. Unter dieser Voraussetzung konnte z.B. Thomas von Aquin das künstliche Gedächtnis der rhetorischen Mnemotechnik (Cicero) mit der psychoiogischen Gedächtnistheorie des Aristoteles fusionieren, um die praktisch-ethische, d.h. exemplarische Funktion der Historie für gegenwärtiges und zukünftiges Handeln zu rechtfertigen. "The aggregation of past phenomena", lautet Colemans einschlägiger Kommentar, "into a meaningful system is coextensive with mind, it is prudence in action" (S.460). Hinter der "rationalen Konstruktion" der Historie (S.455) stand, wie ich ergänzend hinzufügen möchte, die Absicht, Glauben und Wissen zu versöhnen. Denn der Glaube hielt sich an die auctoritas jenes Gedächtnisses, dessen Inhalt sich aus den Exempeln und Lehrsätzen der anerkannten Textüberlieferung zusammensetzte. Diese Überlieferung methodisch zu formalisieren, sie - mit anderen Worten - in die Gewißheit des Wissens zu überführen, war aber Sache der ratio, jenes anderen Grundpfeilers der thomistischen Wissenschaft. Die Texte und Zeichen des Glaubens galten gleichsam als das apriorisch Gegebene. Die Vernunft las darin wie der Leser im Buch, und in dem Maß, in dem sie sich der übernatürlichen Wahrheit assimilierte, steigerte sie die Glaubensdoktrin bis zur Gewißheit einer metaphysischen Erkenntnis.

Colemans historische Recherche schließt mit der Darstellung der im Spätmittelalter einsetzenden Kritik an der thomistischen Harmonisierung von Glauben und Wissen: mit dem Nominalismus der Antiqui (Scotisten) und Moderni (Ockhamisten). Ausführlich erläutert sie die erkenntniskritische Position Ockhams und spricht ihr eine Vorläuferrolle in der Geschichte der neuzeitlichen, der intuitiven Erkenntnis zu. Ockham geht von einer isto statu (nach dem Fall) gegebenen kontingenten Wirklichkeit aus. Diese zu erkennen, ist nicht Sache der nach Maßgabe logischer Universalien verfahrenden intellektuellen Abstraktion. Wirklichkeitserkenntnis ist vielmehr unmittelbar: intuitives Auffassen der konkreten Einzeldinge, abhängig von Erfahrung und daher entsprechend fehlbar. Die Zeichen der Sprache, der Rede, der Schrift enthalten nichts von der Substanz dieser Erfahrungen, sind bestenfalls, weil allein in mente, deren unvollkommene Substitution. Entzieht sich die Wirklichkeit der logischen Demonstration, so bleibt dennoch die Sprache Gegenstand logischer Analysen. Es ist eine Philosophie der Differenzen, die dieser, übrigens in sich selbst widersprüchliche Nominalismus propagiert. Denn er trennt entschieden zwischen Glaube und Wissen, zwischen empirischer und linguistischer Erfahrung und nicht zuletzt zwischen der raumzeitlich situierten Praxis und ihrer Repräsentation in schriftlichen (z.B. erzählenden) Texten. Es ist vor allem dieser letzte Punkt, der die Einschätzung und Auslegung des historischen Gedächtnisses berührt, soweit dieses sich aus schriftlichen Zeugnissen zusammensetzt. Erinnert werden nur eigene Erfahrungen. Die schriftlich festgehaltenen vergangenen Erfahrungen anderer, gerade auch die des sakralen Schriftkanons, sind - was für die Erben des historischen Bewußtseins trivial ist - dem späteren Interpreten als solche nicht zugänglich. Und so stößt der Nominalist, der sich auf der Grenze zwischen erlebter und beredeter Welt eingerichtet hat, auf einen weiteren, den Formalismus der scholastischen Textauslegung überwindenden Unterschied; mit Colemans Worten: "the distinction between subject matter and expression, between rhetorical formulation and its object" (S.528). Eine Unterscheidung, die den theologischen Sockel der Kanonpflege unterminiert und bald die historisch-philologische Kritik auf den Plan rufen wird. 
Die nominalistische Anerkennung der Kontingenz und die implizierte Aufwertung der Empirie können als Antworten auf die historischen Erfahrungen des Spätmittelalters - Schwächung des Papsttums und der Idee eines christlichen Imperiums, Pestepidemien und Bauernrevolten, zunehmende Konkurrenz nationaler Interessen etc. - verstanden werden. Innertheologisch wollte der Nominalismus Ockham'scher Prägung aber den Glauben stärken, indem er der weltlichen Unsicherheit ein Gottesbild entgegenhielt, das vom Gläubigen unbedingtes Vertrauen in die ordnende Kraft der Providenz verlangte. Es ist daher verwunderlich, daß Coleman der mit dem Nominalismus gleichzeitigen Reorganisation der praktischen und theoretischen Diskurse im Zeichen der Wiedergeburt (Renaissance) antiken Wissens den innovatorischen Charakter bestreitet. ${ }^{21}$ Sie betont die inhaltlichen Kontinuitäten und interpretiert den Übergang vom Mittelalter zur Renaissance lediglich als "Genrewechsel" (genre shift: S.573ff.).

Nun ist die humanistische Rezeption des Nominalismus sicher unbestreitbar. Ebenso unbestreitbar ist aber auch die Kritik insbesondere der italienischen Frühhumanisten - Petrarca, Coluccio Salutati, Leonardo Bruni u.a. - an der leeren Dialektik der Moderni à la Ockham. ${ }^{22}$ Diese gewiß oft polemische Kritik allein als Ausdruck eines literarischen Formenwandels abzutun, wird dem Paradigmenwechsel, den die humanistische Bewegung einleitete, nicht gerecht. Nur weniges sei hier in Erinnerung gerufen:

Erstens: Grundlegend ist die humanistische Ablösung epistemologischer Interessen durch eine Praxeologie, die den Begriff aktiven Handelns gesellschaftlich definiert. Das allein markiert eine wesentliche Differenz zur nominalistischen Reduktion der Tathandlung auf die inneren Erfahrungen des Individuums.

Zweitens: Mit ähnlicher Stoßrichtung bauen die Humanisten den Gegensatz zwischen Rhetorik und Logik aus. Die von ihnen propagierte Lebensform der vita activa gewinnt erst die politische Gestalt einer vielstimmigen und vielfältigen vita civilis durch öffentliche Rede und Gegenrede. Rede und Wort unterscheiden sich wie der Text vom Lexem. War die nominalistische Logik aufs Wort, auf den "terminus", fixiert, so bedenkt die Rhetorik den ganzen kommunikativen Redeakt unter allen nur möglichen Bedingungen seiner situativen Anwendung und seines intendierten Erfolgs.

Drittens: Die humanistische Erkenntnislehre rückt die Kultur - im weitesten Sinne des Worts - in den Mittelpunkt. Nur das, was der Mensch geschaffen hat, ist auch seinem Erkenntnisvermögen zugänglich. Damit ist eine Aufwertung der Poiesis verbunden, die zugleich das Bewußtsein der kreativen Selbsttätigkeit und der subjektiven Erfahrung zu steigern vermag.

Viertens: Künstlern und Gelehrten der Renaissance wird eine Distanz zwischen Gegenwart und Altertum bewußt, auf die sie mit der Suche nach der unverfälschten, der wahren Erinnerung reagieren. Diese Suche führt sie durch den Zweifel am Prozeß der Überlieferung hindurch auf die Erosionsspuren des Vergessens. ${ }^{23}$ Im Licht des Distanzbewußtseins erscheint den

21. S. Colemans Kritik an den Thesen Peter Burkes: op. cit., S.563ff.

22. Vgl. P. Kondylis: Die neuzeitliche Metaphysikkritik, Stuttgart 1990, S.45ff.

23. Dazu S. Settis: "Continuità, distanza, conoscenza. Tre usi dell'antico", in: S. Settis (Hg.): Memoria dell'antico nell'arte italiana, Bd.III, Turin 1986, S.373-486. 
Humanisten die Überlieferungs- als Verfallsgeschichte. Diese durch die Wiederherstellung (restauratio) des verlorenen Gedächtnisses zu heilen und das Geheilte auf lebenspraktische Fragen anzuwenden, wird zum Programm einer neuen Mnemonik.

Dennoch, eines scheint sicher: Die theologischen Bemühungen des christlichen Mittelalters haben dem Erinnern und der Gedächtniskunst eine normative Valenz verliehen, die es in den Gesellschaften der griechisch-römischen Antike in dieser Weise vermutlich nicht gab. ${ }^{24}$ Erscheint die Kultur des christlichen Mittelalters dem Historiker unter dem Bild einer "Erzähl- und Erinnerungsgemeinschaft", dann muß er sich fragen, was diese von andern Epochen und andern Kulturen unterscheidet. Denn der mnemonische Sinn der Gemeinde sollte nicht nur - wie Kierkegaard schrieb - "nach rückwärts", sondern auch "nach vorn", auf die Parusie, schauen: ${ }^{25}$ Memoria eschatologica - ein dritter, ein proleptischer Typus des Eingedenkens, dessen Eigensinn sich krass vom spontanen und anamnetischen Erinnern unterscheidet und dennoch - paradoxerweise - an die Botschaft der überlieferten Texte anknüpft. Alltags- und Religionskultur dieser Epoche standen unter dem biblisch-patrimonialen Imperativ: "Tut dies zu meinem Gedächtnis!" Zur Konditionierung eines entsprechenden Kollektivgedächtnisses gehörten das ritualisierte Eingedenken - Eucharistie, Heiligenkult, Kalender und religiöse Feste des Kirchenjahres, Totenmesse etc. - und der ständige Umgang mit einer reichhaltigen schriftlichen, auch piktoralen Gedächtniskunst, die alles, vom Layout der Buchseite bis zur architektonischen Raumgestaltung, im Geist der Mnemotechnik ausgeführt hat. Um einige konkrete Dinge zu nennen: Chroniken, Glossare, Enzyklopädien, Städte- und Länderbeschreibungen, Genealogien, Bilderbibeln, Kirchenfenster, Fresken, Tapisserien; kurz alles das, was sich nach numerischen und topographischen Ordnungsprinzipien organisieren und mit sinnlich wahrnehmbaren Zeichen, Bildern, Symbolen verknüpfen ließ. ${ }^{26}$

Mary Carruthers hat in The Book of Memory die vormoderne Kultur, zumal die des Mittelalters, als eine der Erinnerung von der "dokumentarischen" Kultur der Moderne abgegrenzt. Aber auch sie ist bereit, den mittelalterlichen Memorialismus als ein Kapitel aus der Vorgeschichte des modernen Denkens zu deuten. Assoziiert man versuchsweise, was der wörtliche Sinn der Begriffe nahelegt, mit dem Begriff der Memorialkultur den des Monuments (= Erinnerungszeichen), so rücken die Konzeptionen von Coleman und Carruthers in einen Gegensatz zu Michel Foucaults Konzeption der Kulturanalyse, der zu denken gibt. Foucault hat versucht, dem Erinnerungspathos der Kulturwissenschaften entschlossen den Rücken zu kehren. Denn Erinnerung in dem von ihm inkriminierten Sinn ist das Medium historischer Kontinuitätsbildung, das aus Furcht vor der Brüchigkeit kultureller Normen die Spur eingeschliffener Lesarten und

24. Zur Sakralisierung eines verpflichtenden Kanons durch die Kirche s. Assmann (Anm.13), S.116ff.

25. Zit. nach J. B. Metz: "Erinnerung", in: Handbuch philosophischer Grundbegriffe, hg.v. H. Krings / H. M. Baumgartner / C. Wild, Bd.2, München 1973, S.388. Zahlreiche Bibel-Belege für die imperativische Mnemonik der jüdisch-christlichen Glaubensdoktrin zitiert J. Le Goff: "Memoria", in: Enciclopedia Einaudi VIII, Turin 1979, S.1081ff. Vgl. allgemein J. Le Goff: Histoire et mémoire, Paris 1986.

26. Zur sozialen und politischen Funktion der Gedächtniskultur im Mittelalter s. die Einzelstudien in: Memoria in der Gesellschaft des Mittelalters, hg.v. D. Geuenich und O. G. Oexle, Göttingen 1994. 
Rezeptionen nicht verläßt: Die Kulturwissenschaftler interpretieren die vergangenen Erscheinungen als "Dokumente" eines Sinnzusammenhangs, dessen gesteigerter Form sie selber angehören. Das kulturelle Gedächtnis, so könnte man Foucaults Kritik umschreiben, ist unter diesen Voraussetzungen nichts anderes als ein Zauberspiegel, in dem die kurzsichtige Gegenwart nur den Konstitutionsprozeß ihres Zusichselbstkommens zu entziffern sucht. Es komme aber darauf an, fordert Foucault, das Archiv der Überlieferung mit dem Blick des Ethnologen durchzumustern, der das Vergangene als Diskontinuum und im Eigenen auch das Andere wahrnimmt. ${ }^{27}$

Wie viele andere vergleichbare kulturhistorische Studien verfahren auch die Colemans hermeneutisch, indem sie den subjektiv vermeinten Sinn ihrer Dokumente im Rahmen eines retrospektiv auszumessenden Kontinuums historischer Theorien und Praktiken rekonstruiert. Ihr Gegenstand ist, um es noch einmal anders zu formulieren, ein kulturelles Gedächtnis, dem so geartete Studien angehören und das sie deshalb nur von innen her - eben mit hermeneutischen Mitteln - explizieren können. Nicht die Berechtigung dieses Verfahrens steht hier zur Debatte. Es geht mir vielmehr um die Suche nach einer kulturanalytischen Alternative, die auch das Ausgeschlossene, die vergessenen und verdrängten Diskurse, $\mathrm{zu}$ thematisieren vermag, die das stillschweigend als Dominante kultureller Ordnung vorausgesetzte Gedächtniskonzept auszuschließen droht.

\section{Imitation und Variation als Prinzipien produktiver Erinnerung}

Der Begriff des kulturellen Gedächtnisses scheint eine normative Geltung des zu Erinnernden einzuklagen. Auswahl und Gewichtung dessen, was im Gedächtnis haften soll und die Methode des imitativen Lernens bedingen einander. Quintilian hat in der Institutio oratoria (Buch X.1) einen literarischen Kanon festgeschrieben, aber er hat den Rhetorikschüler nicht nur zur Imitatio, sondern auch zum Wettbewerb (aemulatio) mit den großen Mustern aufgefordert. Die sklavische Nachahmung, ist daraus zu schließen, würde ebenso wie das sklavische Auswendiglernen ein lebendiges Erinnern ersticken. Schon Quintilian spielt, der scholastischen Pedanterie seines Werkes zum Trotz, auf eine Kunstübung an, die der mnemotechnischen Instanz der imitativen Aneigung den Zugang zur Variation nicht versperrt. Vielleicht bedarf es nur einer anderen Lesart, um auf Spielräume in den klassischen Texten zu stoßen, die es nahelegen, die Kulturgeschichte als einen rekursiven Prozeß zu verstehen, in dem Imitation (Kontinuität) und Variation (Wandel) zusammenwirken. Eine Vermutung, die im folgenden an Beispielen aus der Renaissance-Forschung zu überprüfen ist.

Zwischen Kanon und Konvention besteht ein enger Zusammenhang. Dieser steht zur Disposition, sobald ein alter Kanon zugunsten eines neuen verworfen wird. Im spätmittelalterlichen Italien begann der schöpferische Umbau konventioneller kanoni-

27. Zur theoretischen Grundlegung M. Foucault: L'archéologie du savoir, Paris 1969. Paradoxerweise greift Foucault zur Kennzeichnung der wissenschaftlichen Gegenstandskonstitution jenseits von Text- sowie hermeneutisch erschließbaren Sinngestalten (="document") auf den Begriff "monument" zurück, um das Forschungsobjekt aus der konventionell unterstellten geistesgeschichtlichen Dienstleistung zu entlassen. Zu den Schwierigkeiten, mit denen diese Konzeption zu kämpfen hat, vgl. M. Frank: "Ein Grundelement der historischen Analyse: die Diskontinuität - Die Epochenwende von 1775 in Foucaults 'Archäologie'", in: Herzog / Koselleck (Anm. 1), S. 97-130. 
scher Kunstformen zuerst im Bereich der architektonischen Künste. ${ }^{28}$ Vielfältig waren die Motive: Sie schlossen die polemische Abkehr vom Baustil der.Gotik und die Suche nach einer Formensprache ein, die den Bedürfnissen nationaler Identität entsprach. Die Wahl fiel auf die Baudenkmäler und Ruinen der römischen Antike. Mario Carpos Studie Metodo ed ordini nella teoria architettonica erläutert diesen Kanonwechsel an Texten von L. B. Alberti, Raffael, S. Serlio und G. D. Camillo. Sein Ausgangspunkt sind jene Fragen, die mit der Lehrmethode auch die Praxis der Baukunst berühren: In welchem Verhältnis stehen Regeln und Exempel? Welche Möglichkeiten und Grenzen hat die altbewährte Methode der Imitatio? Was nützen Modelle? Was ist eine Komposition? Welche Funktion erfüllt ein Fragment, ein Zitat? Welche Beziehungen bestehen zwischen Theorie und Praxis? Welche zwischen Grammatik, Rhetorik, Topik, Poetik? Alles Fragen, die auch im Kontext anderer zeitgenössischer Lehrsysteme - der Poesie, der Malerei, der Musik - von Bedeutung waren. ${ }^{29}$ Zwar gaben die italienischen $\mathrm{Au}$ toren der Architekturlehre verschiedene Antworten, doch einte sie die Suche nach einer Richtschnur, d.h. einem Kanon, der nicht 'nordischen' (Gotik) oder 'griechischen' (Byzanz), sondern genuin römischen Ursprungs sein sollte. Ein wohlgemeintes Programm, das in der Praxis jedoch zu einem neuen Stil geführt hat, in dem sich ältere und jüngere Bautraditionen überlagern.

Vitruvs zur Zeit des Augustus entstandener Traktat De architectura war auch dem Mittelalter bekannt, zum Kanon avancierte er aber erst in der Traktatliteratur der italienischen Humanisten. ${ }^{30}$ Die humanistischen Kommentare diskutierten vor allem die architektonische, anthropometrisch fundierte Ordnungs- und Proportionenlehre des Römers. ${ }^{31}$ An erster Stelle ist hier - nicht zuletzt wegen der weit über Vitruv hinausführenden Systematik - Leon Battista Albertis De re aedificatoria zu nennen, ein Traktat, dem Carpo das Kapitel "Regole ed ordini" gewidmet hat. Alberti verzichtet in seiner Abhandlung auf jede bildliche Illustration, konzentriert sich auf die von der klassischen Baukunst abgeleiteten Regeln und arbeitet mit Methoden, die teils der scholastischen Traktatliteratur, teils der rhetorischen Inventio nahestehen. Sein Ziel ist nicht der imitierbare visualisierte Kanon, sondern eine architektonische Topik, die dem Benutzer die Freiheit läßt, die abgeleiteten formalen Regeln je nach Gusto in plastische Baukonstruktionen umzusetzen. Hielt sich die frühhumanistische Imitatio naiv an die Betrachtung der "stummen Texte" tradierter exemplarischer Werke, zeigt nun die Theorie, daß diese Werke einem Prozeß rationaler Konzeption und Planung zu verdanken sind (S.44f.). Der humanistische Traktat setzt zunächst auf theoretischer Ebene das Prinzip der Variation gegen die Erstarrung des imitatorischen Konventionalismus und bereitet damit - so paradox das klingen mag - die Entmachtung des soeben erst kanonisierten Vitruvius vor. So empfiehlt Alberti seinen Lesern, nicht nur den

28. Vgl. P. Burke: Tradition and Innovation in Renaissance Italy. A Sociological Approach, [o.O.] 1974, S.340.

29. Zur Verschiebung der ars musica von der mittelalterlichen Zahlenlehre zur humanistischen Poetik, von der imitatio zum ingenium, vgl. K. W. Niemöller: "Zum Paradigmenwechsel in der Musik der Renaissance. Vom numerus sonorus zur musica poetica", in: Literatur, Musik und Kunst im Übergang vom Mittelalter zur Neuzeit, hg.v. H. Boockmann et al., Göttingen 1995, S.187-215.

30. L. A. Ciapponi: "Il 'De Architectura' di Vitruvio nel primo Umanesimo", Italia medievale e umanistica 3 (1960), S.95ff. Vgl. L. Callebat, "La Tradition Vitruvienne au Moyen Age et à la Renaissance. Eléments d' Interprétation," International Journal of the Classical Tradition (IJCT) 1.2 (Fall 1994), S. 3-14.

31. F. Zöllner: Vitruvs Proportionsfigur. Quellenkritische Studien zur Kunstliteratur des 15. und 16. Jahrhunderts, Worms 1987. 
schwerverständlichen Vitruv, sondern auch die sichtbaren Monumente der römischen Baukunst zu studieren. ${ }^{32}$ Der kontrapräsentische Rückgriff auf das 'Gedächtnis' der römischen Architektur - so möchte ich Carpis Befunde verallgemeinern - ist nicht imitatio im Sinne der Wiederholung, sondern methodisch und zugleich durch Anschauung vermittelt. Albertis Traktat ist exemplarisch für diese Spielart der literarisch und wissenschaftlich verfahrenden Erinnerungsarbeit. Ihn interessiert nicht die Zweckgebundenheit der Baukunst, sondern der Möglichkeitssinn verschiedener Lösungen: das Prinzip der varietas "als Ausdruck menschlicher Individualität". 33

Zwischen 1450, dem Entstehungsdatum von De re aedificatoria, und 1544, dem Erscheinungsjahr von Giulio Camillos Trattato dell'imitazione, verschieben sich - wie Carpo zeigen kann - die Schwerpunkte der baukünstlerischen wie auch die anderer Lehrmethoden weiter ins Gebiet wissenschaftlicher Reflexion. Dieser Wandel ist aufs engste mit der Aufwertung der rhetorischen inventio und ihrer Seitendisziplin, der Topik, verknüpft. Wer den literarischen Diskurs über die Konventionen hinaus erweitern will, der imitiert und variiert zugleich auf der Grundlage des Studiums der Quellen und Monumente die klassischen Muster. Imitatio, historische Retrospektion und Modellabstraktionen werden kompatibel; in allen Künsten verwandeln sich die Schablonen (exempla) in Modelle, die Modelle in Bilder (S.35). Carpo beschreibt diesen Wandel als Versuch, die eingeschliffenen Lehrmethoden abzulösen und die Beziehungen zwischen Grammatik, Rhetorik und Dialektik - nicht zuletzt in der Baukunst - pragmatisch zu reorganisieren (S.34ff.). Das Resultat dieser Entwicklung ist zunächst ein Bruch zwischen Norm und Nachahmung, zwischen "System und Exempel" (S.52). Die Normbildung abstrahiert, das Regelsystem generalisiert. Die schöpferische Imitation aber verschreibt sich der Intuition und begünstigt den freien Umgang mit den vorgefundenen Mustern. Es entsteht jener 'abgeleitete Stil' (J. Burckhardt) der Renaissancekunst, der nur von der Architektur zu reden - weltweite Verbreitung finden wird.

Um die mit dieser Entwicklung verbundenen Konflikte zwischen Herkommen und Neuorientierung zu lösen, versuchen sich die Pädagogen und Theoretiker des Cinquecento an einer "wissenschaftlichen" Methode der empirischen Analyse, die Carpo mit seinen Autoren als induktive "tecnica della divisione" bzw. "metodo divisivo" bezeichnet (S.55). Auch diese neue Methode hat ihre Vorformen in Antike und Scholastik. ${ }^{34}$ Aber sie verfährt, anders als diese, weder deduktiv noch logisch-demonstrativ. Eher steht sie jenen empirischen Verfahren der Anatomie nahe, die Niccolò Leoniceno in seinen Galen-Kommentaren von 1508 dargelegt und als methodisch-mnemotechnisches Organon empfohlen hat. ${ }^{35}$ Kern dieses Verfahrens ist die anatomische Zerlegung

32. L. B. Alberti: De re aedificatoria, hg.v. M. Finoli und P. Portoghesi, Mailand 1966, Bd.II, S.441. Vgl. C. Thoenes: "Anmerkungen zur Architekturtheorie", in: Architekturmodelle der Renaissance. Die Harmonie des Bauens von Alberti bis Michelangelo, hg. v. B. Evers, München/New York 1995, S.28-39; H.-W. Kruft: Geschichte der Architekturtheorie. Von der Antike bis zur Gegenwart, München ${ }^{3} 1991$, S.47ff.

33. Kruft (Anm.32), S.49.

34. Zur Anwendung der divisio in der scholastischen Logik und Topik vgl. M. Grabmann: Die Geschichte der scholastischen Methode, Bd.II: Die scholastische Methode im 12. und beginnenden 13. Jahrhundert [1911], Darmstadt 1956, S.426ff., 476ff.; dto. in der mittelalterlichen Sakralkunst W. Kemp: "Visual Narratives, Memory, and the Medieval Esprit du System", in: Küchler / Melion (Anm.13), S.87ff.

35. N. Leoniceno: In libros Galeni e greca in latinam linguam a se translatos praefatio communis, Venedig 1508. Zur Rolle Galens in der Ausbildung methodischen Denkens vgl. N.W. Gilbert: Renaissance Concepts of Method, New York/London 1960, S.3ff. 
(divisio) des Objekts, des Textes oder Baukörpers, in die ihn konstituierenden Einzelteile und deren katalogartige Archivierung. Im Anschluß an analytische Operation und kompilatorische Aufzeichnung werden die Teile nach Art und Gattung bzw. Gebrauchsfunktionen klassifiziert und in Stemmata (arbores scientiae) übersichtlich in Ordnung gebracht. Diese Methode, auch "kurzer Weg" (via brevis) genannt, erweist sich als besonders erfolgreich, da ihr diagrammatisches Endprodukt zum Auge spricht und auf beliebige Objektbereiche anwendbar ist. Bald weit verbreitet und fortschreitend verfeinert, findet sie Anwendung in der Enzyklopädistik, in der Mnemonik Camillos "teatro della memoria" -, in Zitatensammlungen, in der Neuen Logik des Petrus Ramus (58ff.), im Vocabulario der Accademia della Crusca und in Cesare Ripas Iconologia. ${ }^{36}$ Sie ist, wie Carpo betont, eine Antwort auf die Suche nach neuen Kompositionsformen in allen Künsten und für all diejenigen, die, selbst wenn sie kein Talent besitzen, sich an einem literarischen oder bildkünstlerischen Werk versuchen möchten. Denn sie archiviert wie eine Bibliothek oder ein Kunstkabinett das in Teile und Fragmente, kurz: das in zitierbare Einheiten zerlegte Gedächtnis der antiken Überlieferung und stellt es jener ars combinatoria zur Verfügung, die auf das Alte nicht verzichtet, sondern es wie eine Sammlung dekonstruierter Bausteine unter Rahmenbedingungen ausbeutet, die einer Befreiung von den Anweisungen des inhaltlich festgelegten Kanons entsprechen.

Sebastiano Serlio aus Bologna, Autor eines in ganz Europa rezipierten, mächtig nachwirkenden architekturtheoretischen Regelwerks, war mit Giulio Camillo, dem Erfinder jener hermetischen Gedächtniskunst, befreundet, die sich selbst als Universalschlüssel zum Geheimnis des ewigen Kosmos und die irdische Welt als "terra oblivionis" verstand ${ }^{37}$ In einer Vorlesung mit dem Titel L'idea dell'eloquenza hat Camillo auf komplizierte Weise eine doktrinale Rhetorik entwickelt, die eine globale "Architektur des Wissens und des Kosmos" schaffen wollte (S.66). Camillo konstruierte zu diesem Zweck das Denkbild einer siebenstufigen Treppe, über die die Lehrmethode einer jeden Disziplin von den Einzelerscheinungen zu den Prinzipien auf- und von dort wieder hinuntersteigen sollte. Carpo interpretiert dieses methodische Auf und Ab als einen Versuch, zwischen den empirischen Einzelerscheinungen - "ein Repertoire von exemplarischen Texten: Fälle, Individuen, Ereignisse oder Objekte" (S.71) - und den Regeln bzw. Normen einer Wissensdisziplin, hier z.B. der Architekturdoktrin, zu vermitteln. ${ }^{38}$ Die Einzelerscheinungen werden im Lauf dieses Cursus nach einem "metodo divisivo" (Analyse) zerlegt, der von einem gegebenen Klassifikationsgitter ausgeht; in der Baukunst von Punkten, Linien, Oberflächen, Volumen, geometrischen Figuren, Bauordnungen und Typen (S.64). Auf diesen Schritt folgt die Extrapolation der den Phänomenen zugrundeliegenden Normen (Formalisierung). Entscheidend ist die Nutzanwendung dieses Algorithmus in der Produktion neuer rhetorischer Figuren, neuer 'Texturen' und Bauordnungen. Hier zeigt die Methode ihre innovativen Qualitäten. Denn die Applikation der induktiv gewonnenen Normen aufs Rearrangement des

36. Zu den über Carpo hinausgehenden Beispielen vgl. L. Bolzoni: "Il gioco delle immagini. L'arte della memoria dalle origini al Seicento", in: La Fabbrica del Pensiero. Dall'Arte della Memoria alle Neuroscienze [Ausstellungskatalog], Mailand 1989, S.22ff.

37. S. Serlio: Regole generali di architettura sopra le cinque maniere degli edifici, Venedig 1537/1551. $\mathrm{Zu}$ Camillo vgl. P. Rossi (Anm.14) und L. Bolzoni: Il teatro della memoria. Studi su Giulio Camillo, Padua 1984.

38. Zur kosmologischen Bedeutung dieser Transformation der antiken Mnemotechnik im Werk Camillos und Giordano Brunos vgl. P. Rossi (Anm.4) S.15. 
gegebenen und klassifizierten Materials ist eine Bedingung für "neue kompositorische Synthesen" (S.72).

Camillos Lehrmethode des Auflösens (dissoluzione) und des Wiederzusammenfügens (ricomposizione) im Sinne der Neubildung hat im 16. Jahrhundert Widersacher, aber auch Nachfolger gefunden: z.B. in der Poetik Francesco Patrizi, in der Philosophie Petrus Ramus. Der Architekturtheoretiker Serlio hat, wie Carpo im einzelnen zeigt, Camillos Verfahren aufgegriffen und zu einer "Rhetorik des Zitierens" vereinfacht. Ergebnis ist ein "architektonisches Lexikon", das nicht die Termini der Baukunst verzeichnet, sondern die nach Geometrie, Perspektive und Anordnung klassifizierten Formen sakraler wie profaner Architektur. Dieser visualisierte und leicht zu nutzende Thesaurus sollte den Benutzer anleiten, die antiken, als Ruinenfragmente vor Augen liegenden, im "Lexikon" verzeichneten und geordneten Architekturmuster zu memorieren und, je nach Bedarf und Geschmack, permutativ zu neuen Kompositionen zusammenzufügen. ${ }^{39}$ Serlios Suche nach novità hat der manieristischen Variation und Kombination scheinbar inkompatibler Formen den Boden bereitet. Langfristig gesehen hat dieses relativ freie Spiel mit dem kulturellen Gedächtnis eine Wiederherstellung klassizistischer Normen nicht verhindert, sondern begünstigt. ${ }^{40}$

Der hier skizzierte Umbau der Architekturtheorie ist exemplarisch für eine veränderte Einstellung zur Tradition. Denn die Lehrmethode geht von der Wechselbeziehung zwischen imitatio und inventio, zwischen anamnetischem Erinnern und vorausdenkendem Projektentwurf aus. Der klassische Text bzw. das dem entsprechende Architekturfragment gilt gleichsam als Archetyp, als ein zeitresistentes Monument, dessen normative Geltung die Epoche allmählich historisch relativiert, indem es das Wie und Warum untersucht und die Materialien nicht nur konserviert, sondern nach wissenschaftlichen Kriterien neu anordnet. Die benutzerfreundliche Archivierung und systematische Neuordnung dieses kulturellen Gedächtnisses, die von der Reproduktionstechnik der Buchdruckerkunst begünstigt wurde, stand der Suche nach individuellen Stilen nicht im Wege. Im Gegenteil: sie hat es den Künstlern erleichtert, sich in ein freies Verhältnis zur Macht (auctoritas) des Gedächtnisses zu setzen. Denn methodische Zergliederung, Klassifizierung und Archivierung sind geeignet, die Gegenstände in Distanz zu halten, sie nicht nur als imperativische Monumente (Erinnerungszeichen), sondern auch als erklärungsbedürftige Studienobjekte wahrzunehmen. Die Dis$\tan z$ erst macht den Künstler frei. Sie fördert die Lizenz, sich vom Gewicht der kulturellen Tradition zu entlasten, ohne mit ihr brechen zu müssen. Sie kann unter dieser Voraussetzung im kulturellen Gedächtnis aufgehoben werden.

So paradox es klingt: Die Verwandlung der handwerklichen Imitatio in ein propädeutisches Fach der Künstlerausbildung ist zugleich eine Funktion der Standardfestlegung, nämlich der Kanonisierung. Dafür sind typisch: die besonnene Auswahl unter der Masse des Überkommenen, die Klassifizierung (jedes Muster eine Klasse für sich) und Exklusivität ("Schließung" des Kanons). ${ }^{41}$ Nur das Beste, betonen die Lehrmeister immer wieder, ist nachahmenswert und daher im artistischen Gedächtnis aufzubewahren. Mnemotechnik, Selektion, Reflexion und Phantasie müssen daher - wie Mary

39. S. auch die Studie von L. Olivato: "Dal teatro della memoria al grande teatro dell'architettura: Giulio Camillo e Sebastiano Serlio", Bollettino del C.I.S.A. 21 (1979), S.233-252.

40. Vgl. zur weiteren Entwicklung Kruft (Anm.32), S.80ff.

41. Jan Assmann (Anm.13, S.107) unterscheidet vier Gebrauchsweisen des Kanonbegriffs: "Maßstab, Richtlinie, Kriterium; Vorbild, Modell; Regel, Norm; Tabelle, Liste". 
Pardo in einer Studie über Leonardo da Vincis Lehrmethode zeigt - zusammengehen. ${ }^{42}$

Das "visuelle Gedächtnis" des Schülers der Malkunst hat in den Renaissancetheorien den Status einer Kontrollinstanz. Es hält während des imitativen Studiums der kanonisierten Modelle das fest, was in der bildenden Kunst der Schrift am nächsten kommt: die Zeichnung (disegno) der Körper und Figuren. Doch Leonardo geht es nicht um die bloße Reproduktion. Er empfiehlt dem Schüler das Studium und die Imitatio verschiedener Meisterwerke, um ihn zu eigener Urteilsbildung zu nötigen (Pardo, S.53ff.). Der entscheidende Schritt auf dem Weg zur Meisterschaft aber ist die Anschauung, das Kopieren nach der Natur. Hier endlich bleibt das Gedächtnis hinter dem Vermögen der Einbildungskraft (imaginatio) zurück, die Bewegung der wahrgenommenen Dinge zu registrieren und vor das innere, bildschaffende Auge zu rücken (S.62ff.). Und auch dieses Vermögen stößt noch an Grenzen, wenn es um das höchste Ziel der Malerei geht: die freie und dennoch organische Komposition der Bewegungsfiguren auf dem Tableau.

Leonardos Methode besitzt - das zeigt Pardos Studie - einen eminent reflexiven Zug, der die aristotelische Gedächtnispsychologie in ihre Schranken weist, so als wolle die neue Methode sich von jener moralischen Kontrolle der Phantasie befreien, die im Mittelalter das Zusammenspiel zwischen memoria und imaginatio beherrscht hat. Der Aufstand gegen diese Tradition hat den Maler und Ingenieur immer wieder veranlaßt, den Bedingungen künstlerischer und technischer Kreativität auch auf materiellem, lies: physiologisch-anatomischem Boden nachzuforschen. Wie in den pädagogischen Theorien der Baukunst spielt auch hier die kritische Lektüre der medizinischen und naturphilosophischen Schriften Galens eine katalysatorische Rolle. ${ }^{43}$ Sie hat offenbar jene Methodologie bestärkt, die auf induktivem Weg von der - sei es formalen, sei es empirischen - Analyse der Einzelphänomene über die Prinzipien der Synthesis zu einem veränderten Bild der Wirklichkeit fortschreiten sollte. Doch nicht der Szientismus ante litteram ist das Bemerkenswerte an dieser Entwicklung, vielmehr der Versuch, das visuelle Gedächtnis in eine innerweltliche Kosmosvorstellung zu integrieren. So entspricht in Leonardos Theorie die kompositorische Synthesis im Malakt einem komplizierten Zusammenspiel zwischen Auge, Hand und Seelenvermögen, dessen leitende Idee Harmonie heißt und das sich in letzter Instanz der Erklärung entzieht. Das Ziel, die Harmonie-Idee bildlich zu realisieren, gibt der Malerei die Berechtigung, sich an die Stelle der rhetorischen Mnemonik zu setzen, um deren Beschränkungen zu überwinden. Sie selber erhebt nun den Anspruch auf eine Kunst der Erinnerung, die das sinnlich Wahrgenommene in einen selbstgeschaffenen Kosmos übersetzt und zugleich vom Betrachter eine eigenständige, nämlich ästhetische Rezeptionshaltung verlangt (S.68). Die Figur des Künstlers erscheint in dieser Perspektive bereits als Demiurg einer von ihm geschaffenen diesseitigen neuen Welt, die sich kategorisch vom Gedächtnis der alten Weltbilder unterscheidet.

Das Veralten tradierten Wissens kennzeichnet einen Bruch mit der Überlieferung, der in der frühen Neuzeit verschiedene Antworten herausgefordert hat. Das "advancement of learning" (Francis Bacon), Motor der naturwissenschaftlichen Theoriebildung und experimentellen Praxis im 16./17. Jahrhundert, führt zu einer positiven Bewer-

42. M. Pardo: "Memory, Imagination, Figuration: Leonardo da Vinci and the Painter's Mind", in: Küchler / Melion (Anm.13), S.47-73.

43. M. Kemp: "Il concetto dell'anima in Leonardo's Early Skull Studies", Journal of the Warburg and Courtauld Institutes 34 (1971), S.129ff. S. auch M. Pardo, op. cit., S.220 (Anm.). 
tung des Vergessens und zur Polemik gegen Bücher- und Traditionswissen. Aber wie so oft war auch diese Polemik nichts anderes als eine rhetorische Verallgemeinerung, die das übersah, was Paolo Rossi als einen komplizierten, die Renaissancekultur insgesamt charakterisierenden Rapport zwischen "riscoperta degli antichi" und "senso del nuоvo" beschrieben hat. ${ }^{44}$ Eine Verknüpfungsstelle zwischen Altem (antiquitas) und Neuem liegt gewiß in jener für die Moderne typischen Neugier, die Welt im ganzen der menschlichen Erkenntnis verfügbar zu machen. In ihr liegt letztenendes auch ein Motiv dafür, die Antike nicht nur restaurativ wiederherzustellen, sondern sie als die Ausprägung einer scheinbar vollendeten Kultur $\mathrm{zu}$ bewahren, $\mathrm{zu}$ deuten und $\mathrm{zu}$ erklären. Das ist eben nicht dasselbe wie jene "Rezeption", als deren locus classicus die komplette Überschreibung eines eigenen durch ein anderes, nämlich durch das römische Rechtssystem gilt. Die methodische Aneignung der Antike durch die Humanisten und Renaissancekünstler ist schon Aus-einander-Setzung, deutende Um-Schreibung und anamnetische Arbeit im Namen jener "kontrapräsentischen Erinnerung", die nicht eine, sondern mehrere Traditionen unterscheidet und eine Wahl trifft. Der europäische Humanismus entschied sich gegen das Mittelalter und für die Antike, eine Entscheidung, die in nachhaltiger Weise auch die noch lebendigen mittelalterlichen Traditionen umstrukturiert hat. Nichts belegt besser die Wechselbeziehung zwischen Assimilation und Distanznahme als die von dieser Epoche ausgehende historische Periodisierung, die zwei historische Vergangenheiten - Altertum und Mittelalter - mit der Gegenwart einer neuen Zeit konfrontiert.

Ein Begleiterscheinung der Renaissance war die Illusion, die Ungleichzeitigkeit zwischen Altertum und Gegenwart durch einen Akt der Wiedergeburt überwinden zu können. In Wahrheit stellt dieser Akt die Ungleichzeitigkeit überhaupt erst her. Historisierend vertieft die anamnetische Erinnerung die Unterschiede zwischen inkompatiblen Lebenswelten und ruft Formen der Vermittlung hervor, die - in the long run in die bewahrende und auslegende Arbeit der Kulturwissenschaften einmünden.

\section{Eine Theorie des "kulturellen Gedächtnisses"}

Die Metapher der Wiedergeburt zitiert den Tod, und dieser den dunklen Grund, den die Mnemotechnik zu überbrücken sucht. So jedenfalls erzählt es die Gründungslegende, die Ciceros De oratore dem Gedächtnis der europäischen Literaturen überliefert hat. ${ }^{45}$ Liest man diese Erzählung als metaphorischen Kommentar, so

44. P. Rossi (Anm.5), S.164.

45. Cicero: De oratore, II.86.352ff.: Dicunt enim, cum cenaret Crannone in Thessalia Simonides apud Scopam fortunatum hominem et nobilem cecinissetque id carmen, quod in eum scripsisset, in quo multa ornandi causa poetarum more in Castorem scripta et Pollucem fuissent, nimis illum sordide Simonidi dixisse se dimidium eius ei, quod pactus esset, pro illo carmine daturum; reliquum a suis Tyndaridis, quos aeque laudasset, peteret, si ei videretur. Paulo post esse ferunt nuntiatum Simonidi, ut prodiret; iuvenis stare ad ianuam duo quosdam, qui eum magno opere evocarent; surrexisse illum, prodisse, vidisse nemimen: hoc interim spatio conclave illud, ubi epularetur Scopas, concidisse; ea ruina ipsum cum cognatis oppressum suis interisse: quos cum humare vellent sui neque possent obtritos internoscere ullo modo, Simonides dicitur ex eo, quod meminisset quo eorum loco quisque cubuisset, demonstrator unius cuiusque sepeliendi fuisse; hac tum re admonitus invenisse fertur ordinem esse maxime, qui memoriae lumen adferret. ("Man erzählt nämlich, Simonides habe zu Krannon in Thessalien bei Skopas gespeist, einem reichen und vornehmen Mann, und dort ein diesem gewidmetes Gedicht vorgetragen, in dem er - wie das so Dichterart ist - um der Ausschmückung willen auch viele Worte über Castor und Pollux verlor. Daraufhin habe 
kommt eine eigentümliche Konjunktion zwischen Traditionsbruch - versinnbildlicht im zusammengestürzten Versammlungshaus - und Totengedenken - versinnbildlicht in den mithilfe der Sitzordnung (loci) wiedererinnerten Bildern (imagines) der unter den Trümmern begrabenen Mitglieder der Festgemeinde - ans Licht. Das etwa durch innere oder äußere Katastrophen verschuldete plötzliche Dahinschwinden einer älteren Kulturformation und des mit dieser identischen Wertehorizonts stellt jede Gemeinschaft vor die Frage, auf welche Weise die Gegenwart mit den Erfahrungen und dem Wissen der untergehenden Welt umgehen soll. Eine Frage, die, weit über den bloß technischen Rahmen der Mnemonik hinaus, kulturtheoretische Probleme sehr allgemeiner Art berührt.

"Die ursprünglichste Form, gewissermaßen die Ur-Erfahrung jenes Bruchs zwischen Gestern und Heute," heißt es in Jan Assmanns großer, interdisziplinär und kulturvergleichend angelegter Untersuchung Das kulturelle Gedächtnis, "in der sich die Entscheidung zwischen Verschwinden und Bewahren stellt, ist der Tod."46 Assmann begreift das Weiter- oder Nachleben der oder des Toten (im personalen wie kollektiven $\operatorname{Sinn}$ ) nicht als eine selbstverständliche symbolische Fortexistenz, sondern als einen bewußt gegen das Vergessen inszenierten "Akt der Belebung" durch das Kollektiv. Die Symbole des 'Weiterlebens' müssen erst geschaffen, ihre Verwendungsregeln erst gesetzt oder vereinbart werden. Wo dieses Ineinandergreifen von zeitüberbrückender Mnemotechnik und sozialer Imagination fehlt, läßt sich kaum von Kultur, geschweige denn vom "kulturellen Gedächtnis" reden.

Es ist Assmanns Absicht, diesen Mechanismus der Kontinuitätserzeugung, dem sich nichts weniger als das Identitätsbewußtsein einer Gesellschaft verdanken soll, im Rahmen einer "allgemeinen Kulturtheorie" zu diskutieren (S.19). Im Zentrum dieser Kulturtheorie stehen nicht die simplen didaktischen Regeln der rhetorischen Ars memorativa. Thr Erklärungsobjekt sind vielmehr die komplizierten Relationen zwischen so der Untertitel - "Schrift, Erinnerung und politischer Identität in frühen Hochkulturen", Relationen, die sich im Begriff des "kulturellen Gedächtnisses" überschneiden. In vier beeindruckenden Fallstudien vergleicht Assmann die Leistungen dieses "kulturellen Gedächtnisses" für die "Erfindung des Staates" im alten Ägypten (S.167ff.), für den "Zusammenhang zwischen Recht und Erinnern" in den Keilschriftkulturen (S.231ff.), für die "Erfindung der Religion" in Israel (S.196ff.) und für die "Disziplinierung des Denkens" in Griechenland (S.259ff.). Der Begriff der "Erfindung" verweist schon in der verkürzten Form der Zwischentitel auf den erheblichen Anteil, den Assmanns Theorie dem Imaginaire am Prozeß der Zivilisation zubilligt (vgl.S.133ff.).

der überaus geizige Skopas zu Simonides gesagt, er werde ihm nur die Hälfte des vereinbarten Honorars auszahlen, die andere möge er sich gefälligst von den Tyndariden besorgen, die er zu gleichen Teilen mit Lob bedacht habe. Kurz darauf, heißt es weiter, habe man Simonides gemeldet, er möge vors Haus kommen, es warteten am Tor zwei junge Männer, die ihn dringend sprechen wollten. Er habe sich erhoben und sei hinausgegangen, habe aber niemanden gesehen. Unterdessen sei der Versammlungsraum, in dem Skopas tafelte, eingestürzt und habe ihn mitsamt den Seinigen unter den Trümmern begraben und zugrunde gerichtet. Als die Angehörigen dann die Toten bestatten wollten, hätten sie die verstümmelten Leichen überhaupt nicht identifizieren können. Da soll Simonides, indem er sich den Sitzplatz eines jeden in Erinnerung rief, jeden einzelnen für das Begräbnis bezeichnet haben. Durch dieses Ereignis belehrt, so erzählt man, habe er herausgefunden, daß es vor allem die Ordnung sei, die dem Gedächtnis ein Licht aufsetzt.")

46. J.Assmann (Anm.13), S.33. 
Die erste Hälfte dieses gedankenreichen Buches entwirft in Auseinandersetzung mit dem für Jahrzehnte vergessenen, kürzlich erst wiederentdeckten französischen Soziologen Maurice Halbwachs eine Theorie des kollektiven Gedächtnisses, deren kulturgeschichtlicher Innovationswert in den anschließenden Fallstudien Relief gewinnt. Kritischer Ausgangspunkt ist für Assmann das Nebeneinander zweier moderner Forschungsrichtungen, von denen die eine, die wissenssoziologische Position, den Durchbruch zur Zivilisation im ersten vorchristlichen Jahrtausend aus dem Auftreten neuer intellektueller Eliten und Weltdeutungsmuster erklärt, während die andere, die mediengeschichtliche Position, den Zivilisationsprozeß auf die Evolution der Schriftsysteme zurückführt. ${ }^{47}$ Assmanns Untersuchung will diese Richtungen zusammenführen. Er unterscheidet zu diesem Zweck im Begriff der Kultur zwischen normativen und narrativen Merkmalen: "Regeln und Werte" einerseits, "die Erinnerung an eine gemeinsam bewohnte Vergangenheit" andererseits (S.17). Beide Modi sichern durch Rekurs auf Früheres den Zusammenhalt - in Assmanns Terminologie die "konnektive Struktur" - einer sozialen Ordnung, unterscheiden sich aber von Gesellschaft zu Gesellschaft in der Beschaffenheit der jeweils geltenden kollektiven Erinnerungspraktiken. Diese sind vielfältig, reichen vom mimetischen Handeln über die selbstgeschaffene Dingwelt und den Spracherwerb bis in die institutionellen Räume, in denen sie von Spezialisten auf relative Dauer gestellt werden: Rituale, Denkmäler, Zeremonien, Schrift, Auslegung. Sie bieten, wie die beiden Seiten ein- und derselben Münze, subjektive und objektive Ansichten der "Kultur", insofern sie Sinn und Identität einer kollektiven Lebensform zugleich schaffen und zu sichern imstande sind. Erst mit der mnemotechnischen Organisation der Schriftkultur, so Assmanns Hauptthese, gelingt es aber, die Sinn-Überlieferung von der direkten Kommunikation abzukoppeln. Es entsteht so ein, von der gruppeninternen Sinnzirkulation aus gesehen, materiell-semiotischer Bereich der kontrapräsentischen Bewahrung auslegbarer Vorstellungs- und Bedeutungswelten, der in Kontakt bzw. Konflikt mit den jeweils aktuellen Selbstbildern des Kollektivs treten kann: das kulturelle Gedächtnis. Dieses externe Gedächtnis "speist Tradition und Kommunikation, aber es geht nicht darin auf" (S.23).

Anhand des Kanonbegriffs und der Kanonbildung, um diese zentralen Punkte herauszugreifen, erläutert Assmann die Funktionen des im Medium der Schrift beheimateten kulturellen Gedächtnisses. In einem aufschlußreichen Kapitel rekonstruiert er zunächst die Genese sowie den Bedeutungs- und Funktionswandel des Kanonbegriffs (S.103ff.). Das griechische Wort hatte zunächst mit Schrift nichts im Sinn, bezeichnete es doch das "Richtscheit" oder "Lineal" des Architekten und Baumeisters. Die ursprüngliche semantische Verbindung mit den Maß- und Stabilitätsansprüchen der Tektonik ist noch in der Übertragung auf andere Kunstformen lebendig, etwa in den von Galen, Plinius und zahlreichen andern antiken Autoren überlieferten Kunstregeln des Polyklet und dessen als sicht- und greifbarer "Kanon" rezipiertes Bildwerk Doryphoros. ${ }^{48}$ Eine metaphorische Übertragung des Begriffs auf ethische, politische, artistische u.a. Problemlagen in der antiken Welt ließ nicht lange auf sich warten. Der gemeinsame

47. Für die erstgenannte Position vgl. z.B. S. N. Eisenstadt (Hg.): Kulturen der Achsenzeit. Ihre Ursprünge und ihre Vielfalt, 2 Bde., Frankfurt/Main 1987, für die andere die Arbeiten von E. A. Havelock: Schriftlichkeit. Das griechische Alphabet als kulturelle Revolution, Weinheim 1990, und J. Goody: The Logic of Writing and the Organisation of Society, Cambridge 1986.

48. Vgl. insbes. die Beiträge von E. Berger und H. Philipp in: Polyklet. Der Bildhauer der griechischen Klassik, hg.v. H. Beck et al., Mainz 1991. S. auch W. G. Moon (Hg.): Polykleitos, the Doryphoros, and Tradition, Madison, WI/London 1995. 
Nenner all dieser, über variierende Diskurse zerstreuten Gebrauchsweisen umschließt - mit einem Wort Max Webers - "die Eingestelltheit auf das Regelmäßige" und einen universellen, d.h. situationsabstrakten Geltungsanspruch der zugrundeliegenden Orientierungsfunktionen. ${ }^{49}$

Was die Metaphorisierung andeutet - 'Bau' einer Lebensform nach Maßgabe der tektonischen Richte - das bezieht Assmann verallgemeinernd auf Typen der "Kultur", die ihren Zusammenhalt, ihre Identität, schriftkulturellen Mnemotechniken verdanken. ${ }^{50}$ Wie jede Identität bildet und erhält auch die des Kollektivs sich unter Bedingungen des Ein- und Ausschließens. So kann ein kultureller Kanon in der Gestalt konkreter Normierungen auftreten, die auf einer Skala zwischen den Extremen systematischen Vergessens (Zensur) und fundierender Letztbegründung (Kanon der Vernunft) ihren Ort haben. In jedem Fall zieht der Kanon eine Grenze: zwischen erlaubt und verboten, zwischen heilig und profan, zwischen wertvoll und wertlos, zwischen vernünftig und unvernünftig und verspricht eben dadurch Orientierung. Der Modus wertsetzender Selektion, der das eine aus der Kultur aus-, das andere in sie einschließt, wird in solchen Situationen verschärft, in denen die Auflösung alter Verbindlichkeiten die Gegenwart mit Orientierungslosigkeit bedroht.

Innerhalb der von Assmann kultursemiotisch und -soziologisch erweiterten Grenzen steht "Kanon" als ein abgeleitetes Wertsystem über den selbstverständlichen Normen alltäglicher kultureller Praxis, da das von ihm umschriebene Prinzip ein Ideal (der Vollkommenheit; S.116) kodifiziert, an dem sich die Vergesellschaftung einer Gruppenidentität $\mathrm{zu}$ orientieren hat. Als eine solche "Norm zweiter Ordnung"51 steht "Kanon"

- für die "Grammatik" der spätägyptischen Kultur, verkörpert im Ritus und in der wie ein Buch mit Schrift übersäten monumentalen Tempelarchitektur der Epoche;

- für die hodegetische Mnemotechnik Israels, bezogen auf den Kanon des Kanons und die exegetische Monokultur des geheiligten Textbestandes;

- für die (wissenschaftliche) Disziplinierung agonistischer Strukturen in der Schriftkultur Griechenlands, die - unter den Bedingungen dauernd möglichen Wiederanknüpfens (hypoleptisches Prinzip) an "klassische" Texte zum Medium der Wissensevolution geworden ist.

In allen genannten Hochkulturen, so Assmanns These, antwortet die Kanonbildung auf den Zusammenbruch des 'Hauses' der alten, auf den Fundamenten des Ritus und unbefragter Traditionen gebauten Kultur. Der durch je verschiedene endogene oder exogene Ursachen herbeigeführte Traditionsbruch in der einen oder andern Sozialordnung zwingt diese dazu, um der Selbsterhaltung willen besondere Kompen-

49. M. Weber: Wirtschaft und Gesellschaft. Grundriß der verstehenden Soziologie, Tübingen ${ }^{5} 1972$, S.188.

50. "Die Kanonmetapher postuliert zugleich mit der Konstruktivität der Welt - der Mensch als Baumeister seiner Wirklichkeit, seiner Kultur und seiner selbst - die Letztinstanzlichkeit und Hochverbindlichkeit der Prinzipien, denen solche Konstruktion sich unterwerfen muß, wenn das 'Haus' Bestand haben soll." (Assmann, op.cit., S.127)

51. Zur Definition des Kanons als "Norm zweiter Ordnung" s. D. Conrad: "Zum Normcharakter von 'Kanon' in rechtswissenschaftlicher Perspektive", in: A. und J. Assmann: Kanon und Zensur, München 1987, S.46-61. 
sationsstrategien und Deutungsmuster zu entwickeln. Das führt nicht zur bloßen Erhaltung der Tradition, vielmehr zu ihrer Aufhebung in ein neues Selbstbild, das Assmann als eine "Steigerungsform kollektiver Identität" interpretiert (S.134ff.;193). Die Tradition wird "reflexiv"; will sagen: sie wird in der Bedeutung kontrapräsentischer Erinnerung in Distanz gebracht, und es entsteht jene "symbolische Sinnwelt", die es erlaubt, kategorisch zwischen der "naturwüchsigen" und einer Kultur zweiter Ordnung zu unterscheiden. ${ }^{52}$ Die Kultur zweiter Ordnung ist gegenüber den naturwüchsigen Formationen durch institutionell gesicherte und symbolisch vermittelte Praktiken der Integration, im Sinne der Identitätsbildung, und Distinktion, im Sinne einer Abgrenzung nach außen, ausgezeichnet. Beide Funktionen manifestieren sich in den Organisationsstrukturen eines schriftkulturellen Gedächtnisses, das über den Traditionsbruch hinweg Kontinuität zu bewahren sucht, ohne die Erfahrung des Bruchs wegzuzaubern. "In Israel ist es die Erinnerung einer dissidenten Gruppe [...], die sich im Zeichen der Distinktion auf die Torah gründet. Die zentrale Erinnerungsfigur ist die Geschichte einer Auswanderung, einer Sezession, einer Befreiung aus der Fremde. In Griechenland ist es die gemeinsame Erinnerung vieler zerstreuter Gruppen, die sich im Zeichen der Integration auf die Ilias stützt. Die zentrale Erinnerungsfigur ist die Geschichte einer Koalition, eines panhellenischen Zusammenschlusses gegen den Feind im Osten" (S.273).

Wohl ist es ein Kennzeichen der Kanonbildung, zugleich mit der Kodifizierung des Gedächtnisses auf der Grundlage eines "fundierenden Textes" oder Textcorpus die Autorität des Kanons durch Schließung sicherzustellen. Die Frage ist jedoch, warum ein solcher Kanon über die Zeit seiner Fixierung hinaus Autorität bewahrt. Auf diese Frage gibt Assmann zwei Antworten. So erläutert er u.a. am Beispiel der Homerischen Epen, daß diese selbst teilhaben an der Reorganisation der Kultur, indem sie einer vom Untergang bedrohten Gesellschaft, der des "heroischen Zeitalters", Gestalt und Stimme geben. Diese Epen sind nicht nur, wie es zugespitzt heißt, "Rekonstruktion von Vergangenheit". Es gelingt ihnen auch, die "Summe der Überlieferung in ein Werk völlig neuen Typs ein[zu]bringen" (S.275). Die Vergangenheit wird an der Schwelle ihrer Auflösung, so könnte man zusammenfassend sagen, nicht nur erinnert, sondern im Prozeß der Textualisierung auch erschaffen. Sie wird zur interpretierten Vergangenheit, die das Stigma der Umbruchszeit trägt, deren in der formalen Gestalt des Gedichts gelungene Überwindung selbst noch in späteren Jahrhunderten als ein vollendetes Sinnzeichen in Erinnerung gerufen werden kann.

Assmanns zweite Antwort erläutert die Erinnerung an Homer in der Perspektive der "Klassik" und des "Klassizismus". Diese Erinnerung setzt im 4. Jahrhundert v.u.Zt. ein, in der Zeit eines Traditionsbruches, der alle Mittelmeerkulturen ergreift. Die darauf antwortende Reorganisation des kulturellen Gedächtnisses orientiert sich - wie Assmann vermutet (S.277) - eher an östlichen als an griechischen Modellen der schriftzentrierten Mnemotechnik. War die "große Tradition" der Homerischen Epen im 6. Jahrhundert ein Produkt "zeremonieller Kommunikation" (rhapsodischer Vortrag im Rahmen der panathenäischen Spiele und panhellenischen Feste), so begründen nun die alexandrinischen Philologen eine "Buch- und Lesekultur", in deren Zentrum der "professionalisierte" Umgang mit Texten, nicht zuletzt mit dem kanonisierten Korpus der

52. Ich folge hier nicht Assmanns Sprachgebrauch, sondern übernehme und variiere den Begriff "zweiter Ordnung" von Y. Elkana: "Die Entstehung des Denkens zweiter Ordnung im antiken Griechenland", in: Eisenstadt (Anm.47: Bd.1, S.52ff.). 
Homer-Epen steht. ${ }^{53}$ In diesem Milieu entfaltet sich "eine neue und andere Kultur, die von Alexandria aus auf die griechische zurückblickt. Die Welt der Literatur scheidet sich in 'die Alten' (hoi palaioi, antiqui) und 'die Neuen' (hoi neoteroi, moderni), und es ist die Dialektik der Innovation, die das Altertum konstituiert" (S.278). Hier steht "Altertum" für eine philologisch und mnemotechnisch auf Dauer gestellte Überlieferung, die vergangen ist und dennoch als "klassische" dem historischen Relativismus die Stirn bieten kann. Diese Entwicklung begreift Assmann als Innovation, weil sie auf der Grundlage kultureller Kontinuität über den Traditionsbruch hinweg die soziale Kohärenzbildung von rituellen Praktiken löst, um an ihre Stelle literarische Praktiken zu setzen: der Text, nicht die Schrift, rückt in den Mittelpunkt der Kulturgeschichte. Das kulturelle Gedächtnis wird damit frei für die oben beschriebenen Prozesse des Wiederanknüpfens (Hypolepse) und der Variation jener Inhalte, die es in Form situationsunabhängiger Texte aufbewahrt. Jetzt erst sind die Bedingungen geschaffen, um jene literarischen Kanones zu fixieren, denen das Prädikat "klassisch" zugesprochen werden kann. Das gilt es festzuhalten: Nicht die Rezeption im Sinne des Aneignens, sondern der aus der Kulturkrise geborene Akt erinnernden Wiederanknüpfens entscheidet über die Gestalt dessen, was als universell anschlußfähiger, nämlich "klassischer" Textkanon in Geltung bleibt.

Was in Alexandria geschieht, hat eine fast zeitgleiche Parallele in der hebräischen Kultur. Wird in der griechischen Kultur Homer zum "Kristallisationskern" des Klassikerkanons, so spielt dort die Torah eine vergleichbare Rolle (S.279). Beide Entwicklungen stehen in Kontakt, und beide schaffen die Bedingungen für jenes zeitresistente textuelle Gedächtnis, an das spätere Epochen um der Kontinuität willen wieder anknüpfen werden: das kulturelle Gedächtnis des säkularen "Abendlandes" an die griechische Klassik, das der Christen und Muslime an die hebräische Bibel (S.280). Dieser Schritt vom Geltungsbereich der gelebten Tradition zur bewußten Stiftung eines literarischen Kanons als Medium soziokultureller Neuorientierung verläuft gleichwohl in den genannten Kulturen unterschiedlich. Denn in Griechenland besteht nicht der von der Religion ausgehende Zwang zur Festschreibung eines einzigen, von Priestern überwachten, widerspruchsfreien Kanons. Hier begünstigt die Literarisierung vielmehr die Gesetze der streitbaren Auslegung, die nicht nur zur weiteren Kulturdifferenzierung, sondern auch zur Ausbildung autonomer kritischer Diskurse beitragen. Die nun entdeckte Möglichkeit, kritisch auszuwählen, setzt ein kodifiziertes, institutionell eingehegtes Wissen voraus; mit anderen Worten: ein mnemotechnisch organisiertes "Gedächtnis", das sich im Medium von literarischen Klassifizierungen, intertextuellen Beziehungen und ästhetischen (nicht religiösen) Diskursen entfalten kann. Zwar verändert allein schon das Aufschreiben den präsentischen Zeithorizont, da es die Möglichkeit schafft, durch Auswahl und Kanonisierung die Gegenwart an dieser oder jener im Gedächtnis der Schrift aufbewahrten Vergangenheit teilhaben zu lassen. Doch erst die von den Griechen entwickelte Mnemotechnik hat die absolute Verbindlichkeit des kanonisierten Textbestandes abgebaut und die Kanonbildung dem Wandel unterworfen. Seitdem sind die Geltungsgründe traditional legitimierter Sinnbestände wie diese selber offen für Kritik. Das vom Klassikerkanon Ausgeschlossene fällt nicht dem kategorischen Imperativ der damnatio memoriae zum Opfer, sondern steht, sozusagen abgeschattet, für künftige Renaissancen zur Verfügung (S.121).

Die Wirkungen dieser Innovation, resümiert Assmann, dauern an und bestimmen

53. Zum Begriff der "großen Tradition" vgl. R. Redfield: Human Nature and the Study of Society, Chicago 1962. 
"die konnektive Struktur unserer eigenen Kultur, das kulturelle Gedächtnis der westlichen Welt, bis heute" (S.300). Die oben beschriebenen Untersuchungen über die mittelalterlichen und humanistischen Anknüpfungen an die antike Literatur scheinen dem Recht zu geben. Sie könnten darüber hinaus Anlaß sein, die These vom Traditionsbruch als Ausgangspunkt für eine Reorganisation des kulturellen Gedächtnisses an den europäischen Gesellschaften des 12. Jahrhunderts und der frühen Neuzeit zu überprüfen. ${ }^{54}$

\section{Medien des Gedächtnisses und kulturwissenschaftliche Erinnerungsarbeit}

Assmanns allgemeine Kulturtheorie korrigiert mit guten Gründen die Auffassung, schon die Evolution bestimmter Schriftsysteme habe einen kulturellen Wandel herbeigeführt. ${ }^{55}$ Der Kulturbegriff bezeichnet in seiner Konzeption die Organisation jenes Wissens, das die Identität gesellschaftlicher Formationen garantiert. ${ }^{56}$ Dieses, etwa in der Form des Mythos narrativ, in kanonischen Texten präskriptiv codierte Wissen verschafft der Gruppe den "Gemeinsinn", auf den sie ihre Selbstdefinitionen stützen kann (S.140ff.). Eine Bedingung sind entsprechende Formen öffentlicher Repräsentation: Inszenierungen im Medium ritueller, zeremonieller oder textzentrierter Handlungen. Diese Formen bedürfen der Institutionalisierung und der Herausbildung kulturtragender Eliten, die zur Produktion, Reproduktion und Zirkulation des sinnstiftenden Wissens beitragen. Je komplexer eine Gesellschaft, desto stärker die Tendenz zur sozialen Differenzierung zwischen kultur-repräsentativer Elite und Kollektiv, so daß nicht nur von einer integrativen, sondern auch von einer dissoziierenden Funktion der Kultur die Rede sein muß. Es bedarf daher, um die identitätsbildende Kraft der "Kultur" zu sichern, ihres Heraustretens aus der "habitualisierten Selbstverständlichkeit" (S.151). Sie muß objektiv werden im materiellen Sinn der Verfügbarund Sichtbarkeit. Und genau diese objektivierende Fixierung bezeichnet der Begriff des "kulturellen Gedächtnisses", der freilich die grundsätzliche Ambivalenz der so "integrativ gesteigerten Kultur" nicht aufhebt, da auch diese zur Produktion von nach außen wirkenden Feindbildern eingesetzt werden kann (S.124ff.).

Assmanns Entwurf rückt Kultur und Gedächtnis eng zusammen. Die beiden Zentralbegriffe - "Kultur" und "Gedächtnis" - sind keine historischen, sondern theorieabhängige Konzepte, die den Vorteil haben, daß sie nicht nur passive, sondern auch aktive Kompetenzen umschreiben. ${ }^{57}$ Die altehrwürdige, auf antike Quellen zurückge-

54. Vgl. zum 12. Jh. C. H. Haskins: The Renaissance of the 12th Century [1927], New York 1961; R. L. Benson / G. Constable (Hg.): Renaissance and Renewal in the Twelfth Century, Cambridge, MA 1982 [ND Toronto 1991].

55. Vgl. auch Assmanns Hinweise auf analoge Entwicklungen in den asiatischen, auf nichtalphabetische Schriftsysteme gebauten Kulturen (etwa S.148ff.)

56. Hier ist kritisch anzumerken, daß die Identitätsbildung nicht nur auf symbolisch strukturierten Kontexten beruht, sondern auch von den je spezifischen Organisationsformen der Arbeit (Ökonomie) und der Herrschaft (Politik) in einer Gesellschaft abhängig ist.

57. Merkwürdig ist die versteckte Beziehung zwischen Assmanns kulturhistorischen Paradigmen (monumentale Kultur Ägyptens/bewahrend-verehrende jüdische Kultur/wissenschaftlich-kritische Kultur Griechenlands) und den drei historischen "Betrachtungsarten" Nietzsches in Vom Nutzen und Nachteil der Historie fiur das Leben: monumentalische/antiquarische/kritische Historie; "Betrachtungsarten", die dazu beitragen sollten, unter Wiederanknüpfung an einen Kanon der Meisterwerke die Produktivkräfte der Kultur im Sinne der 
hende Auffassung vom Magazin oder Speicher des Gedächtnisses wird zwar nicht ganz aufgegeben, aber entschieden erweitert. "Erinnern" steht im Rahmen der von Assmann skizzierten mnemotechnischen Organisation für eine bedeutungs-, ja kulturstiftende Kompetenz, deren "griechische" Variante das Wechselspiel zwischen Kontinuität und Wandel ausdrücklich einschließt.

Problematisch erscheint mir indessen die Verbindung ritueller Identitätsstiftung mit dem Zwang zur möglichst "abwandlungsfreien Wiederholung" (S.89). In Assmanns Theorie bildet diese den Gegenbegriff zu jener "Textualität" des schriftkulturellen Gedächtnisses, die durch variierendes "Wiederanknüpfen" immer wieder - um im Bildfeld zu bleiben - neue, wenn auch nicht fremde Webmuster möglich macht. Die Mnemotechniken eines kulturellen Systems schließen jedoch die mechanische Repetition aus, versteht man sie - wie ich vorschlagen möchte - generell als ein Zusammenwirken zwischen Anamnese und Mnemosyne. Rituelle Handlungen mit symbolischer Konnotation sind selten als bloße Wiederholungen gedacht. Vielmehr bestätigen sie 'als wäre es das erste Mal' - die kollektive Ordnung, indem sie, Initiationsriten sind ein prominentes Beispiel, auf paradoxe Weise die Dialektik von Anarchie und sozialer Kontrolle zur Darstellung bringen. In der Aussageperspektive philosophischer Verallgemeinerung: "la répétition est la différence sans concept." 58

Der begriffsfernen, nämlich bildzentrierten und rituellen Konstruktion soziokultureller Erinnerung haben Susanne Küchler und Walter Melion ein interdisziplinäres Projekt gewidmet, dessen Ziel eine "kulturübergreifende Gedächtnistheorie" ist. Im Einleitungskapitel des von ihnen edierten Sammelbandes Images of Memory, der philosophische, kunsthistorische und ethnologische Studien zusammenfaßt, erläutern sie den interdisziplinären Rahmen ihres Forschungsprogramms. In dessen Mittelpunkt steht die Frage, welche Formen und Funktionen die über Generationen geübte Weitergabe (transmission) von Bilddarstellungen in verschiedenen Kulturen zeigt. Methodisch gesehen sollen bildliche Repräsentationen auf die spezifischen Handlungskontexte der einzelnen Kulturen bezogen und die Funktionen sowohl der "Kanonbildung" (kunsthistorische Perspektive) als auch der "sozialen Kohäsion" (ethnologische Perspektive) in das Spiel einer wechselseitigen Auslegung gebracht werden. Ausgangspunkt ist die Hypothese, daß durch "image production" die Wechselbeziehung zwischen Erinnerungsprozeß und Kulturentwicklung nachhaltig gesteigert werde (S.2ff.). An diesem komplizierten Prozeß interessiert die hier zu Wort kommende Forschung nicht in erster Linie die durchgehaltene Topik, sondern die bedeutungsbildende Differenz, die in der Transmission der visuellen Artefakte innerhalb eines kulturellen Systems aufbrechen kann.

Dieses Forschungsprogramm wendet sich entschlossen vom passiven Gedächtnismodell ab, um folgende Prämissen zu statuieren (S.7):

1. "memory" bezeichnet eine soziokulturelle Konstruktion;

2. es operiert dynamisch und entfaltet seine aktive Kraft über das Medium bildlicher Repräsentationen;

3. alle Formen kollektiven Erinnerns (recollection) sind historisch bedingt und daher von dem, der sie verstehen will, zu kontextualisieren;

Remythologisierung wiederzubeleben. Vgl. zu Nietzsche meinen Essay "Kritik der Geschichte im Namen des Lebens. Zur Aktualität von Herders und Nietzsches geschichtstheoretischen Schriften", Archiv fuir Kulturgeschichte 68 / 2 (1986), S.436ff.

58. G. Deleuze: Différence et répétition, Paris ${ }^{4} 1984$, S.36. 
4. Vergessen und Erinnern sind als korrelative Funktionen zu betrachten, da das kollektive Gedächtnis um der soziokulturellen Bestimmtheit (Standardisierung) willen eine Wahl treffen muß, die das Nicht-Relevante ausschließt resp. methodisch vergißt.

Der Katalog lenkt den Blick auf die Unterschiede und Gemeinsamkeiten zwischen den kunsthistorisch-anthropologischen und den oben abgehandelten historisch-philologischen Forschungsperspektiven. Gemeinsam ist beiden die Betonung der aktiven, der bedeutungs-, kulturkonstitutiven und vergesellschaftenden Funktionen kollektiven Erinnerns im Rahmen eines kulturhistorischen Erklärungsmodells, das Kontinuität und Wandel bedenkt. Indessen gewichtet die amerikanische Forschungsgruppe stärker die innerhalb der Tradierungsprozesse auftretenden Verschiebungen, die historischphilologische Richtung (Coleman; Assmann) stärker die kontinuitätsstiftenden Funktionen. Am auffallendsten aber ist die unterschiedliche Wahl der Erinnerungs-Medien: hier die Schrift-, dort die Bildkultur. ${ }^{59}$

Man geht sicher nicht fehl, wenn man die mnemonischen Funktionen der visuell wahrnehmbaren Artefakte und Praktiken (Ritual, Tanz) im Zivilisationsprozeß genauso hoch einschätzt wie die Evolution der Schriftkultur. Und doch liegt es auf der Hand, daß diese Funktionen sich mit dem Übergang von einem schriftlosen zu einem schriftkulturellen System entscheidend verändern. Festrituale, in deren Zentrum Bildwerke oder Tänze stehen, regulieren den Wechsel zwischen sozialer Distanz und Partizipation naturgemäß in weitaus direkterer Weise als der wie immer szenische Vortrag (Lesung, Schauspiel) von Texten, die auch unabhängig von der direkten Kommunikation aktualisiert werden können. Das zeigen auch die Untersuchungen in Images of Memory. Auf der einen Seite stehen die innerhalb einer entwickelten Schriftkultur entstandenen Bilder mittelalterlicher, neuzeitlicher und chinesischer Malerei ${ }^{60}$ In diesen Fällen ist die produktive Spannung zwischen Schrift und Bild nicht zu übersehen. Nicht nur die handwerkliche Bildherstellung, auch die Formen und Leistungen sowohl des visuellen Gedächtnisses als auch der symbolischen Kodifizierung sind in diesen Fällen in ähnlicher Weise über Texttraditionen, Musterbücher und Lehrsysteme (z.B. der Rhetorik und Hodegetik) vermittelt wie der kontrapräsentische Kanonwandel, der die Erfindung der Renaissancearchitektur begleitet hat (s.o.S.428ff). Auf der anderen Seite stehen jene Untersuchungen, die sich solchen mnemonischen Funktionen szenischer und zugleich bildlicher Darstellungen widmen, die dem von Assmann beschriebenen Typus der "rituellen" oder "zeremoniellen Kommunikation" zuzurechnen sind. ${ }^{61}$ In diesen Fällen beruhen die bildlichen Herstellungsprozesse und rituell aktualisierten Funktionen auf mündlicher Tradierung und auf der unmittelbaren kinästhetischen Partizipation des Kollektivs im Moment der Aufführung. Das kollektive

59. Assmann geht kurz auf die Bedeutung der Bilder als Medium des kulturellen Gedächtnisses Ägyptens ein, erkennt aber in der Evolution des spätägyptischen Schriftsystems den entscheidenden "Innovationsschub" (op.cit., S.192,265f.).

60. W. Kemp: "Visual Narratives, Memory, and the Esprit du System" (S.87ff.); M. Pardo: "Memory, Imagination, Figuration: Leonardo da Vinci and the Painter's Mind" (S.47ff.); W. Melion: "Hendrick Goltzius" (S.8ff.); R. Vinograd: "Private Art and Public Knowledge in Later Chinese Painting" (176ff.).

61. S. Küchler: "Malangan" (S.27ff.); A. L. Kaeppler: "Memory and Knowledge in the Production of Dance" (S.109ff.); G. Feeley-Harnik: "Finding Memories in Madagaskar" (S.121ff.); A. G. Miller: "Transformations of Time and Space: Oaxaca, Mexico, circa 1500-1700" (S.141ff.). 
Gedächtnis kennt hier nicht die räumliche und zeitliche Distanz zu einer Vergangenes dokumentierenden und doch immer wieder aktualisierbaren, weil latent gegenwärtigen Textwelt. Es konstitutiert sich vielmehr nach dem Rhythmus der vom rituellen Kalender vorgeschriebenen performances über die Modi der sinnlich-körperlichen Erfahrung.

Mir scheint, daß die hier verglichenen Konzepte einander nicht ausschließen, sondern ergänzen. Natürlich ist Assmann historisch im Recht, wenn er mit der entwickelten Schriftkultur eine neue Form der kulturellen Mnemotechnik heraufziehen sieht. Im Rahmen einer allgemeinen Kulturtheorie beanspruchen andererseits auch diejenigen Formen der Gedächtnisbildung einen festen Ort, die mit visuellen, ja allgemein sinnlichen Erfahrungen in Zusammenhang stehen. ${ }^{62}$ Soll der Satz "Durch Erinnerung wird Geschichte zum Mythos." (Assmann, S.52) Allgemeingültigkeit beanspruchen, so muß "Erinnerung" - wie das einer vormodernen rhetorischen Tradition entsprach - mit der bildschaffenden Kraft, mit der Imagination, zusammengedacht werden. Denn die zeichenschaffende Arbeit der Imagination ist es, die eine bloße Reproduktion des in die Gegenwart gerufenen Inhalts verhindert. Unter dieser Voraussetzung ist Erinnerung schon "eine Stufe der 'Reflexion'", da sie die Inhalte "als etwas Vergangenes und dennoch für [das Bewußtsein] selbst nicht Verschwundenes im Bilde vor sich hinstellt." 63 Die kognitive Semantik geht noch darüber hinaus. Denn sie behauptet - Mark Johnson weist darauf hin - die Fundierung bildschematischer Wahrnehmungsmuster in körperlichen Erfahrungen und begreift diese sogar als Substrat für alle Leistungen symbolischer und intellektueller Abstraktion. ${ }^{64}$ Von dieser Seite bedarf, wie mir scheint, Assmanns Kulturtheorie der Ergänzung. Immerhin ist die Bildwelt Griechenlands zu einem Anknüpfungspunkt für die europäische Kunst- und Kulturentwicklung geworden, der der antiken Schriftkultur mindestens ebenbürtig ist.

Die Erinnerungsarbeit der historisch-philologisch verfahrenden Kulturwissenschaften, das mögen die hier diskutierten Forschungserträge gezeigt haben, ist Teil der Kultur, die sie erklärt. So decken sich weitgehend die von Assmann an den frühen Umbruchstellen der okzidentalen Kulturgeschichte abgelesenen Prinzipien der Textualität, des hypoleptischen Wiederanknüpfens und der kontrapräsentischen Erinnerung mit den noch heute in den historisch-philologischen Disziplinen gültigen Normen. Gewiß, unter den Bedingungen der modernen Medienrevolution und transkulturellen Überschneidungen verstehen sich diese Disziplinen nicht mehr als Hüter oder gar Erneuerer des Kanons. ${ }^{65}$ Und dennoch weben sie - eine Kultur dritter Ordnung - unter An-

62. Vgl. etwa die Versuche, über Bildinterpretationen einen Zugang zur Fremdheit der griechischen Kultur zu gewinnen, in: C. Bérard, J.-P. Vernant et al. (Hg.): La cité des images, Lausanne 1984.

63. E. Cassirer: Philosophie der symbolischen Formen, Bd.I: Die Sprache, Darmstadt 1973, S.23.

64. "Image schemata that are grounded in our bodily experience are the basis for metaphorical and metonymic mappings by which we understand various nonphysical, abstract domains, such as those of mental processes and epistemic relations." M. Johnson: "The Imaginative Basis of Meaning and Cognition", in: Küchler / Melion, op. cit., S.85. S. auch H. Weinrich: "Über Sprache, Leib und Gedächtnis", in: Materialität der Kommunikation, hg.v. H. U. Gumbrecht, K. L. Pfeiffer, Frankfurt/Main 1988, S.80-93.

65. "Aus den Horizonten normativer und formativer Wertsetzungen kommen wir nicht heraus", bemerkt Assmann und weist den "historischen Wissenschaften" die Aufgabe zu, die Kanon-Grenzen ins Bewußtsein zu rufen (op. cit., S.129). Wie weit sein Ansatz trägt, zeigt auch das von ihm in der Harvard University Press angekündigte Buch Moses the Egyptian: 
wendung der genannten Prinzipien weiter an jenem Textkontinuum, das dem kulturellen Gedächtnis der westlichen Gesellschaften zugrunde liegt und tragen die Erinnerung an die "klassische Tradition" weiter.

Gibt es eine Alternative? Michel Foucault hat sie, denke ich, mit dem Hinweis auf den ethnologischen Blick angedeutet. Die kulturanthropologischen Studien in Images of Memory haben nicht das Gedächtnis der eigenen, sondern fremder Kulturen zum Gegenstand. Sie sind weniger der Geschichtsschreibung als der sozialwissenschaftlichen Beobachtung verpflichtet und können daher mit dem Kontinuitätsparadigma der historisch-philologischen Wissenschaften wenig anfangen. Überträgt man, was Foucaults Hinweis nahelegt, diese Einstellung auf die Erinnerungsarbeit an der eigenen Kultur, so hat das Folgen, die auf eine Verfremdung des habituell verfestigten kulturellen Gedächtnisses hinauslaufen. Die "klassische Tradition" erscheint dann nicht mehr als selbstverständliche Gegebenheit, und die Geschichtsschreibung nähert sich dem, was Paul Veyne einmal die "Wissenschaft der Differenzen" genannt hat. ${ }^{66}$ Ein Perspektivwechsel dieser Art ist möglich, wenn die theoretische Fiktion des unendlich anschließbaren Textkontinuums gegen die theoretische Fiktion eines zwar unüberschaubaren, aber an jeder beliebigen Stelle zugänglichen Symbol-Gewebes ausgetauscht wird. ${ }^{67}$ Textualität ist im Rahmen der "interpretive anthropology", auf die ich hier anspiele, nicht schriftkulturell definiert, sondern Metapher für die analytische Lesbarkeit diverser kultureller Praktiken im Kontext sozialen Handelns - gewissermaßen Kritik am konventionellen Textmodell durch Erweiterung seiner Grenzen. Die Unterscheidung zwischen Kultur und Gesellschaft legt den Grund für eine differenzierende Interpretation, die von der Spannung, nicht der Kongruenz beider Institutionen ausgeht. Auch methodisch kehrt die "interpretive anthropology" die Verfahren der historisch-philologischen Konvention um, indem sie nicht von Traditionen, sondern von dem ausgeht, was Clifford Geertz "local knowledge" nennt. Unter dieser Bedingung fällt der ethnologische Blick zunächst auf das mikrologische Detail des zu beschreibenden Textmusters, um, von dort ausgehend, nach und nach befremdliche Zusammenhänge zu entdecken, für die das Pathos der "Großen Tradition" blind ist.

Die hier angedeutete Alternative mag eines deutlich machen: Sie nimmt nicht Abschied von jenen Errungenschaften der mediengeschichtlichen Revolution, die Jan Assmann am Beispiel der achsenzeitlichen Hochkulturen beschrieben hat. Lesbarkeit und Textualität liegen auch ihrem Selbstverständnis zugrunde, werden aber mit dem Ziel der Verfremdung umkodiert. Die Alternativen liegen demnach, so möchte ich schließen, im kulturellen Gedächtnis selbst. Sie müssen nur gesucht werden.

An Essay in Mnemohistory, dessen Inhalt er jüngst im Heidelberger »Gesprächskreis für Kulturanalyse« vorgetragen hat.

66. P. Veyne: L'inventaire des différences, Paris 1976.

67. C. Geertz: The Interpreation of Cultures. Selected Essays, New York 1973. Ders.: Local Knowledge. Further Essays in Interpretive Anthropology, New York 1993. 